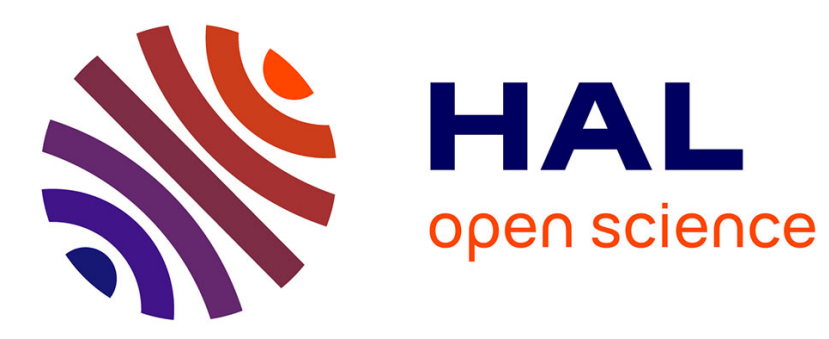

\title{
Robust Energy-aware Routing with Redundancy Elimination
}

\author{
David Coudert, Alvinice Kodjo, Truong Khoa Phan
}

\section{To cite this version:}

David Coudert, Alvinice Kodjo, Truong Khoa Phan. Robust Energy-aware Routing with Redundancy Elimination. Computers and Operations Research, 2015, 64, pp.21. 10.1016/j.cor.2015.05.008 . hal01155639

\section{HAL Id: hal-01155639 \\ https://inria.hal.science/hal-01155639}

Submitted on 27 May 2015

HAL is a multi-disciplinary open access archive for the deposit and dissemination of scientific research documents, whether they are published or not. The documents may come from teaching and research institutions in France or abroad, or from public or private research centers.
L'archive ouverte pluridisciplinaire HAL, est destinée au dépôt et à la diffusion de documents scientifiques de niveau recherche, publiés ou non, émanant des établissements d'enseignement et de recherche français ou étrangers, des laboratoires publics ou privés. 


\title{
Robust Energy-aware Routing with Redundancy Elimination*
}

\author{
David Coudert ${ }^{1,2}$, Alvinice Kodjo ${ }^{2,1}$, and Truong Khoa Phan ${ }^{3}$ \\ ${ }^{1}$ Inria, France \\ ${ }^{2}$ Univ. Nice Sophia Antipolis, CNRS, I3S, UMR 7271, 06900 Sophia Antipolis, France \\ ${ }^{3}$ University College London, UK
}

May 26, 2015

\begin{abstract}
Many studies in literature have shown that energy-aware routing (EAR) can significantly reduce energy consumption for backbone networks. Also, as an arising concern in networking research area, the protocol-independent traffic redundancy elimination (RE) technique helps to reduce (a.k.a compress) traffic load on backbone network. Motivation from a formulation perspective, we first present an extended model of the classical multi-commodity flow problem with compressible flows. Moreover, our model is robust with fluctuation of traffic demand and compression rate. In details, we allow any set of a predefined size of traffic flows to deviate simultaneously from their nominal volumes or compression rates. As an applicable example, we use this model to combine redundancy elimination and energy-aware routing to increase energy efficiency for a backbone network. Using this extra knowledge on the dynamics of the traffic pattern, we are able to significantly increase energy efficiency for the network. We formally define the problem and model it as a Mixed Integer Linear Program (MILP). We then propose an efficient heuristic algorithm that is suitable for large networks. Simulation results with real traffic traces on Abilene, Geant and Germany50 networks show that our approach allows for $16-28 \%$ extra energy saving with respect to the classical EAR model.
\end{abstract}

Keywords: Robust Network Optimization; Green Networking; Energy-aware Routing; Redundancy Elimination.

\section{Introduction}

Recent studies have shown that Information and Communications Technology (ICT) is responsible for $2 \%$ to $10 \%$ of the worldwide power consumption $[25,16]$. For example, the Global e-Sustainability Initiative estimated the overall network energy requirement for European telecommunication is around 35.8 TWh in 2020 [13]. To this extend, the backbone networks and more precisely IP routers, consume a majority of energy [5]. While the traffic load has only a marginal influence, the most contribution of energy consumption on router is the number of active elements such as ports, line cards, base chassis [32]. Traditionally, networks are always designed to meet the peak-hour traffic demand. Therefore during normal periods, the traffic load is typically well below the network capacity. Following this observation, people have proposed energy-aware routing (EAR) to minimize the number of used links while all the traffic demands are routed without any overloaded links [16, 27, 40, 24, 33].

${ }^{*}$ This work has been partially supported by ANR project Stint under reference ANR-13-BS02-0007, ANR program "Investments for the Future" under reference ANR-11-LABX-0031-01, and by the Région PACA. 
Another research topic that has also been active recently is traffic redundancy elimination (RE) $[2,3,37,41]$. Observing that traffic on the Internet contains a large fraction of redundancy (e.g. popular contents such as new movies are often downloaded several times subsequently), a complementary approach uses redundancy elimination (RE) techniques to reduce link load in backbone networks. It consists in splitting packets into small chunks, each is indexed with a small key, that are cached along traffic flows as long as they are popular. Then, keys are substituted to chunks in traffic flows to avoid sending multiple times the same content, and the original data are recovered on downstream routers based on the cache synchronization between the sending and the receiving routers. Therefore, traffic redundancy is removed and traffic volumes of flows between the two routers are reduced. For simplicity, a traffic flow from which redundancy has been removed is called a compressed flow. We use interchangeably the notation compression rate or $R E$ rate to denote how much traffic redundancy can be eliminated.

From energy saving perspective, $\mathrm{RE}$ has a drawback since it increases energy consumption of routers [23]. To find a good trade-off, in our previous work, we proposed GreenRE - a model that combines EAR and RE to increase energy efficiency for backbone network [23]. In the GreenRE model, each of the demand has a static traffic volume and is associated with a constant factor of redundant traffic. To handle future changes and guarantee a certain level of quality of service (QoS) (avoiding overloaded links), the peak volumes of traffic demand and the lowest RE rates are used as the worst case realization. Such assumption clearly leads to inefficient usage of network resources and poor energy saving. To alleviate this limitation of the GreenRE model, the uncertainty on traffic volumes and RE rates has to be precisely modeled and taken into account in the optimization process. By using this extra information, we are able to obtain a design which is closely related to the dynamics of the traffic pattern, hence significantly increase energy saving compared to previous proposals.

In mathematical literature, the technology-independent $\Gamma$-robustness has been introduced in $[8,9]$ and then successfully applied to various network design problems [30, 18, 17]. This approach is based on an observation that in real traffic traces, only a few of the demands are simultaneously at their peaks. So, the authors considered a parameter $\Gamma>0$ so that at most $\Gamma$ demands deviate simultaneously from their nominal traffic volumes. Based on this assumption, the so-called robust solution is a solution that is feasible for any subsets of $\Gamma$ demands simultaneously at their peaks, other demands are being at their nominal values. The originality of the method is the expression of the maximum sum of deviation over all possible subsets of $\Gamma$ demands as a unique linear program (LP). However, this LP formulation may have an exponential number of constraints. To overcome this issue, the LP formulation is transformed into a compact one using the duality theorem.

In this work, we first present an extended version of the classical multi-commodity flow problem in which traffic flows can be compressed to smaller volumes (with some costs, e.g. energy cost mentioned in this paper). Previous studies have considered robustness either on traffic volumes [30] or on redundancy rates [18]. To the best of our knowledge, this is the first work combining uncertainties in both traffic demand volumes and compression rates. In summary, we make the following contributions:

- From a theoretical point of view, we present an extended multi-commodity flow problem with compressible traffic flows. In addition, we provide a robust model in which uncertainties in both traffic volumes and compression rates are taken into account.

- This extended model can be applied to a vast range of applications in network flows and traffic management. In this paper, as an applicable example, we use this model to increase energy efficiency for a backbone network. We apply this extended model 
into energy-aware routing and formally define the Robust-GreenRE problem using Mixed Integer Linear Program (MILP).

- Since robust network design is NP-hard problem [10], we propose a heuristic algorithm that is effective for large instances.

- By simulation, we show energy saving offered by our methods on backbone networks with real-life data traffic traces and compression rate fluctuations.

\section{Related Works}

\section{$2.1 \quad$ Energy-aware Routing (EAR)}

EAR aims at using network protocols to control the whole network, so as to minimize energy consumption while preserving QoS requirements. Before going into details of EAR, we first present an energy profile of a router from a traffic load point of view. An energy profile is defined as the dependence of the energy consumption of a router on its traffic load. In fact, there are several energy profiles in which different functions are used to describe the relationship between energy consumption and traffic load on router [36]. In this section, we present the two main energy profiles: "Idle Energy" and "Fully Proportional" models (Fig. 1).

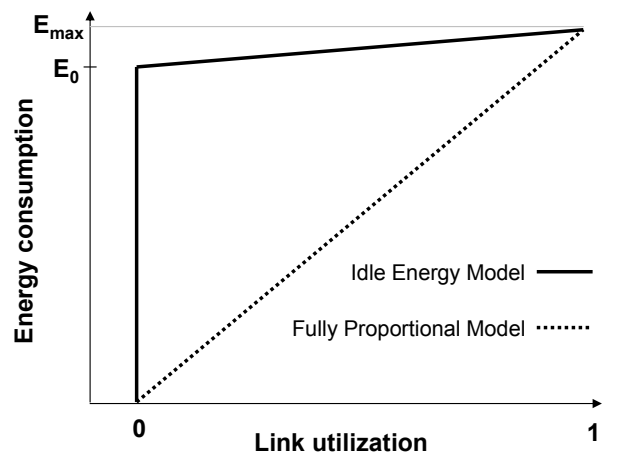

Figure 1: Energy profiles

- Fully Proportional Model: this model represents an ideal case where energy consumption varies linearly with the device utilization, between 0 and $E_{\max }$. As stated in [11], network devices could present such a behavior if techniques like Dynamic Voltage Scaling (DVS), modular switching fabrics, etc. are applied to the components of the devices. Furthermore, the authors in [34] have proposed methods to build a power-proportional software router. Such a model is desired in green networking. However, today network devices are not power-proportional, and it is considered as a futuristic scenario.

- Idle Energy Model: this model is also named "on/off" energy profile. It has been shown in [14] that the energy consumption of today network equipments is not proportional to the quantity of the transported traffic. In practice, network device's energy consumption grows linearly between a minimum value $E_{0}$ and a maximum value $E_{\max }$ which correspond respectively to the idle state and the maximum utilization state (Fig. 1). For more details, a database of power consumption values for ICT network equipments is presented in [28].

In this paper, we focus on the "Idle Energy model" to design and evaluate efficient energyaware routing (EAR) protocol. We refer the reader to $[20,21]$ for more general studies on 
energy-aware problem (with different energy profiles). In our work, the most basic notion of EAR includes mechanisms for turning off or putting components into sleep mode. In general, networks are designed with redundant links and over-provisioning bandwidth to accommodate traffic bursts as well as to allow rerouting when links fail. As a result, the networks are underutilized most of the time, leaving a large room for energy saving. Intuitively, it is possible to have multiple paths between a pair of source-destination nodes in a network. When traffic load is low, we can aggregate the traffic into a few links so that other links do not carry any traffic. Then, idle links of routers can be put into sleep mode for saving energy. In fact, turning off entire routers can earn significant energy saving. However, it is difficult from a practical point of view as it takes time for turning on/off and also reduces life cycle of devices. Therefore, like existing works $[15,23]$, we assume to turn off (or put into sleep mode) only links to save energy.

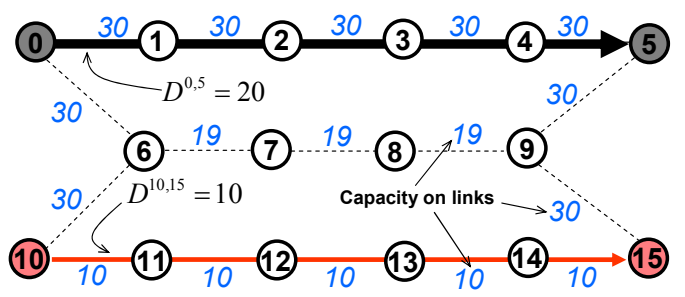

(a) SPR: sleep 7 links

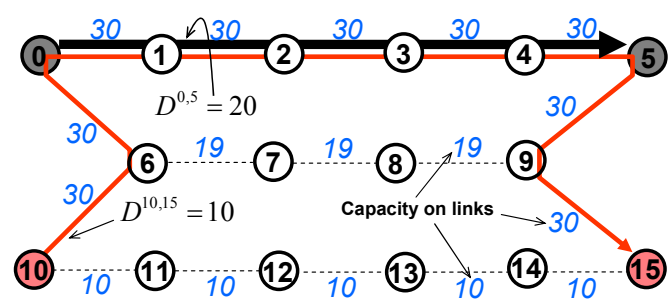

(b) EAR: sleep 8 links

Figure 2: Example of Shortest Path Routing (SPR) vs. Energy-aware Routing (EAR)

As an example of EAR, we refer to Fig. 2. There are two traffic demands $0 \rightarrow 5$ and $10 \rightarrow 15$ with volumes $D^{0,5}=20 \mathrm{Gbps}$ and $D^{10,15}=10 \mathrm{Gbps}$. The shortest path routing, as shown in Fig. 2a, uses 10 active links whereas the remaining 7 links can be put into sleep mode. However, taking energy consumption into account, in Fig. 2b, EAR solution allows to put 8 links into sleep mode, thus energy consumption is further decreased. The problem of minimizing the number of active links under QoS constraints can be precisely formulated using MILP. However, this problem is known to be NP-Hard [22], and currently exact solutions can only be found for small networks. Therefore, many heuristic algorithms have been proposed to find admissible solutions for large networks [16, 22].

\subsection{Redundancy Elimination}

Internet traffic exhibits a large amount of redundancy when different users access the same or similar contents. Therefore, several works $[38,2,3,4,37,41]$ have explored how to eliminate traffic redundancy on the network. Spring et al. [38] developed the first system to remove redundant bytes from any traffic flows. Following this approach, several commercial vendors have introduced Wide Area Network Optimization Controller (WOC) - a device that can remove duplicate content from network transfers $[12,26,39]$. WOCs are installed at individual sites of small Internet Service Providers (ISPs) or enterprises to offer end-to-end RE between pairs of sites. As shown in Fig. 3, the patterns of previously sent data are stored into the databases of the WOCs at both the sending and the receiving sides. The technique used to synchronize the databases at peering WOCs can be found in [26]. Whenever the WOC at the sending side notices the same data pattern coming from the sending hosts, it substitutes the original data with a small signature (encoding process). The receiving WOC then recovers the original data by looking up the signature in its database (decoding process). Because signatures are only a few bytes in size, sending signatures instead of actual data gives significant bandwidth saving. 


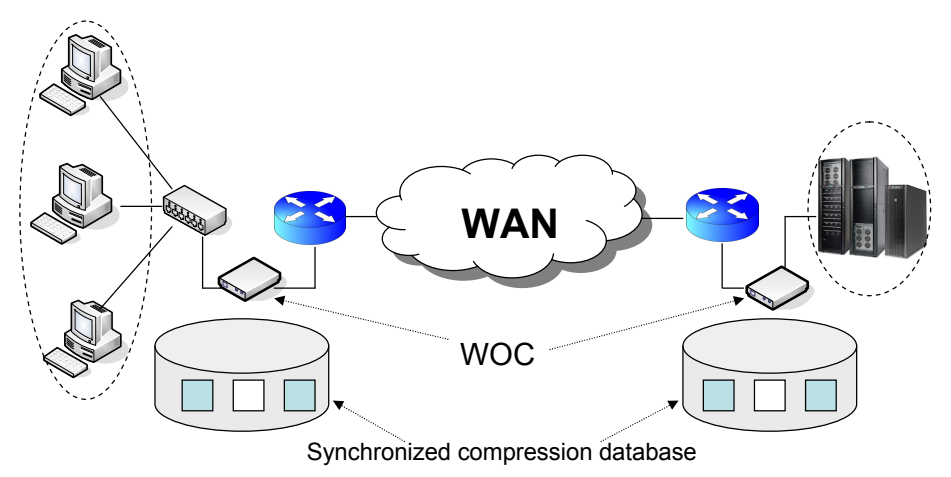

Figure 3: Reduction of end-to-end link load using WOC

Recently, the success of WOC deployment has motivated researchers to explore the benefits of deploying $\mathrm{RE}$ in routers across the entire Internet [2, 3, 4, 37]. The core techniques used here are similar to those used by the WOC: each router on the network has a local cache to store previously sent data used to encode and decode data packets later on. Obviously, this technique requires heavy computation and large memory for the local cache. However, Anand et al. [4] have shown that on a desktop equipped with a $2.4 \mathrm{GHz}$ CPU and 1 GB RAM, the prototype can work at 2.2 Gbps and 10 Gbps respectively for encoding and decoding packets. Moreover, they believe that higher throughput can be obtained if the prototype is implemented in hardware. Several real traffic traces have been collected to show that up to $50 \%$ of the traffic load can be reduced with RE support [3, 4, 37].

In next sub-section, we recall the GreenRE model - the first model of energy-aware routing with RE support [23]. Although RE was initially designed for bandwidth saving, it is also interesting for reducing the network power consumption.

\subsection{GreenRE - Energy Saving with Redundancy Elimination}

In the GreenRE model, RE is used to virtually increase capacity of the network links. However, as shown in [23], a drawback is that when a router performs RE, it consumes more energy than usual. This introduces a trade-off between enabling RE on routers and putting links into sleep mode. We show that it is a non-trivial task to find which routers should perform RE and which links should be put into sleep mode to minimize energy consumption for a backbone network.

\subsubsection{Example of GreenRE model}

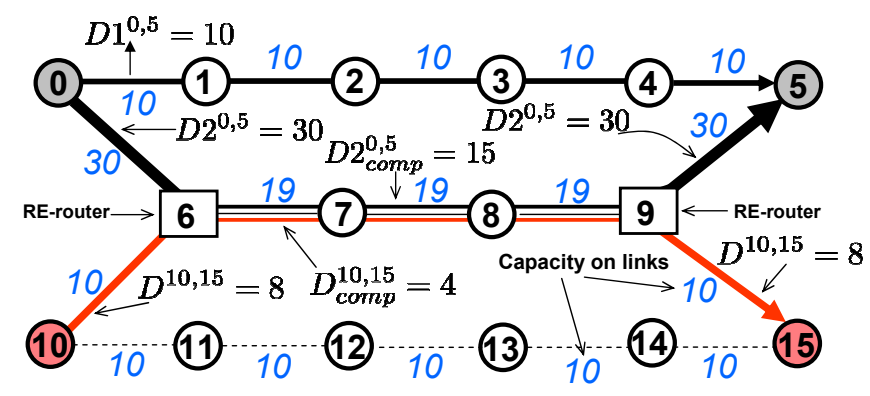

Figure 4: GreenRE with 50\% of traffic redundancy

The example presented in Fig. 4 has two traffic demands $D^{0,5}=40$ Gbps and $D^{10,15}=8$ 
Gbps. As shown in literature [16, 30], the traffic for a commodity can be split among multiple paths between a source and a destination. In the example in Fig. 4, because there is not enough capacity to carry the whole demand $D^{0,5}$ on a unique path, it has to be split into $D 1^{0,5}=10$ Gbps and $D 2^{0,5}=30$ Gbps. Let a RE-router consume 30 Watts [23] and a link consume 200 Watts [16]. Assume that $50 \%$ of the traffic is redundant and RE service is enabled at routers 6 and 9 , thus the traffic flows $0 \rightarrow 5$ and $10 \rightarrow 15$ passing through links $(6,7,8,9)$ are reduced to $15 \mathrm{Gbps}$ and $4 \mathrm{Gbps}$, respectively. As a result, the GreenRE solution allows to put 5 links in sleep mode and to enable 2 RE-routers which saves $(5 \times 200-2 \times 30)=940$ Watts. Observe that in this case, it is impossible for the classical EAR (without redundancy elimination) to find a feasible solution because there is not enough capacity on links to carry the demand $D^{0,5}$ (only 29 units of flows can be routed from node 0 to node 5).

\subsubsection{Problem Formulation}

We consider a communication backbone network where nodes represent routers with multiple interfaces that are used to create physical links. The GreenRE problem is defined on an undirected graph $G=(V, E)$ where $V$ is a set of routers and $E$ represents a set of links. In this network, any physical link between two routers is a bi-directional link, one direction is for the down-stream and the opposite direction is for the up-stream. We use the notation $\{u, v\}$ to denote a physical link (without direction) and $(u, v)$ as an arc with direction from $u$ to $v$. A link $\{u, v\}$ is considered to be active if there is data going through at least one of its directions. Each active link $\{u, v\}$ and router $u$ is respectively associated with a power consumption value $P E_{\{u, v\}}=200$ Watts [16] and $P N_{u}=30$ Watts [23]. We are given a set $\mathcal{D}=\left\{\left(s, t, D^{s t}\right) \in V \times V: s \neq t\right\}$ representing the traffic demands, where $D^{s t}$ denotes the volume of demand from $s$ to $t$. Let $\gamma^{s t} \in[0,1)$ be a compression rate of the demand $\left(s, t, D^{s t}\right)$. For instance, for a $10 \mathrm{Gbps}$ traffic demand with $\gamma^{\text {st }}=0.6$, its volume can be reduced by $\mathrm{RE}$ technique to $10 \times \gamma^{s t}=6$ Gbps of non-redundant traffic. For simplicity, a traffic flow from which redundancy has been removed is called a compressed flow.

We use binary variables $x_{\{u, v\}}$ and $w_{u}$ to denote respectively activated links and RE-routers. $N(u)$ is the set of neighbors of $u$ in the graph $G$. Note that, because all links are bi-directional, all incoming neighbors of node $u$ are also its outgoing neighbors. Variables $f_{u v}^{s t}$ and $g_{u v}^{s t}, \forall\{u, v\} \in$ $E,\left(s, t, D^{s t}\right) \in \mathcal{D}$ denote the fraction of normal and compressed flows $\left(s, t, D^{s t}\right)$ on the arc $(u, v)$ respectively. The notations $C_{u v}$ and $C_{v u}$ represent the capacities of the $\operatorname{arcs}(u, v)$ and $(v, u)$. 
We reformulate the GreenRE model as follows:

$$
\begin{aligned}
& \min \sum_{\{u, v\} \in E} P E_{\{u, v\}} x_{\{u, v\}}+\sum_{u \in V} P N_{u} w_{u} \\
& \text { s.t. } \sum_{v \in N(u)}\left(f_{v u}^{s t}+g_{v u}^{s t}-f_{u v}^{s t}-g_{u v}^{s t}\right)=\left\{\begin{array}{ll}
-1 & \text { if } u=s, \\
1 & \text { if } u=t, \\
0 & \text { otherwise }
\end{array} \quad \forall u \in V,\left(s, t, D^{s t}\right) \in \mathcal{D}\right. \\
& \sum_{\left(s, t, D^{s t}\right) \in \mathcal{D}} D^{s t}\left(f_{u v}^{s t}+\gamma^{s t} g_{u v}^{s t}\right) \leq \mu C_{u v} x_{\{u, v\}} \\
& \sum_{\left(s, t, D^{s t}\right) \in \mathcal{D}} D^{s t}\left(f_{v u}^{s t}+\gamma^{s t} g_{v u}^{s t}\right) \leq \mu C_{v u} x_{\{u, v\}} \\
& \sum_{v \in N(u)}\left(g_{u v}^{s t}-g_{v u}^{s t}\right) \leq w_{u} \\
& \sum_{v \in N(u)}\left(g_{v u}^{s t}-g_{u v}^{s t}\right) \leq w_{u} \\
& 0 \leq f_{u v}^{s t}, g_{u v}^{s t}, f_{v u}^{s t}, g_{v u}^{s t} \leq 1 \\
& x_{\{u, v\}}, w_{u} \in\{0,1\}
\end{aligned}
$$

The objective function (1) is to minimize the power consumption of the network represented by the number of active links and activated RE-routers. Constraints (2) establish flow conservation constraints when considering simultaneously the normal $\left(f_{v u}^{s t}\right.$ and $\left.f_{u v}^{s t}\right)$ and the compressed $\left(g_{v u}^{s t}\right.$ and $\left.g_{u v}^{s t}\right)$ flows. Note that both the normal and the compressed flows can be fractional. The constraints (2) indicate that the sum of flows entering in a router is equal to the sum of flows outgoing from it except if the router is either the source or the destination of the demand. For example, suppose that for a demand $\left(s, t, D^{s t}\right) \in \mathcal{D}$, only normal flow $\left(\sum_{v \in N(u)} f_{v u}^{s t}=1\right)$ enters in a router $u\left(\sum_{v \in N(u)} g_{v u}^{s t}=0\right.$ and $u$ is a RE-router $)$, and there are two outgoing arcs from $u$ that can be used to forward this flow toward its destination node $t:\left(u, v_{1}\right)$ and $\left(u, v_{2}\right)$. Let's assume that another RE-router is available only on the path of the arc $\left(u, v_{1}\right)$ and $50 \%$ of this traffic goes through this link. Therefore only $50 \%$ of the normal flow $\sum_{v \in N(u)} f_{v u}^{s t}$ will be compressed, so half of the compressed flow will be routed through arc $\left(u, v_{1}\right)$. Thus we have: $f_{u v_{1}}^{s t}=0, g_{u v_{1}}^{s t}=0.5$. On the arc $\left(u, v_{2}\right)$ no compression can be made because there is no RE-router on this path. So we have $f_{u v_{2}}^{s t}=0.5, g_{u v_{2}}^{s t}=0$ for half of the normal flow and no compression flow on this arc. This scenario is represented by Fig. 5 where we considered $D^{s t}=50 \mathrm{Gbps}$ and $\gamma^{s t}=0.5$. Consequently the difference of the total incoming flow and the total outgoing flow is equal to 0.

We use constraints (3) and (4) to force the link load to be smaller than the maximum target utilization $^{1} \mu$. Constraints (5) and (6) are used to determine whether RE service is enabled on router $u$ or not. If it is not $\left(w_{u}=0\right)$, the router $u$ only forwards flows without compression or de-compression, then the amount of compressed flows incoming and outgoing the router $u$ is unchanged. It is noted that if a flow is compressed, it needs to be decompressed somewhere on the way to its destination. This requirement is implicitly embedded in the constraints (6). For instance, assume that a destination node $t$ is not a RE-router $\left(w_{t}=0\right)$. When a compressed flow $g_{v t}^{s t}$ reaches its destination, because $t$ is the last node on its path, the flow can not be decompressed. Considering the constraints (6) in that case at node $t$ (i.e., when $u=t$ ), we have $\sum_{v \in N(t)} g_{v t}^{s t}>0$ (the compressed flow enters node $t$ ) and $\sum_{v \in N(t)} g_{t v}^{s t}=0(t$ is the destination

\footnotetext{
${ }^{1}$ This value is provided by the operators, for instance $\mu$ can be set to $50 \%$.
} 


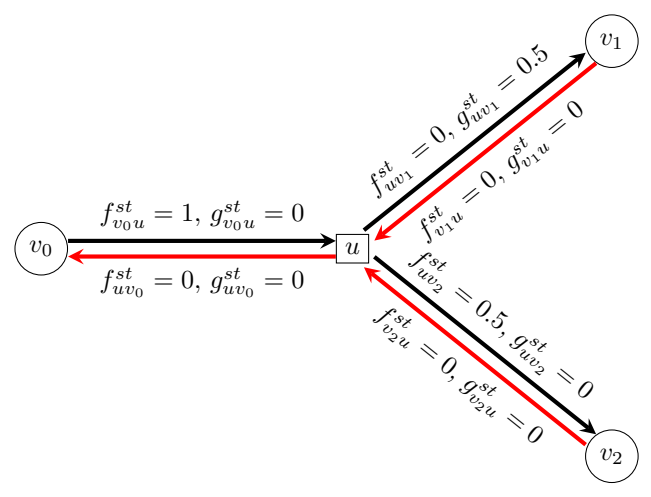

\begin{tabular}{|c|c|c|}
\hline Arc & Normal flow & Compressed flow \\
\hline$\left(v_{0}, u\right)$ & 50 & 0 \\
\hline$\left(u, v_{0}\right)$ & 0 & 0 \\
\hline$\left(v_{1}, u\right)$ & 0 & 0 \\
\hline$\left(u, v_{1}\right)$ & 0 & 12.5 \\
\hline$\left(v_{2}, u\right)$ & 0 & 0 \\
\hline$\left(u, v_{2}\right)$ & 25 & 0 \\
\hline
\end{tabular}

Normal and compressed flow on arcs, in Gbps

Figure 5: Flow conservation with $50 \%$ of traffic redundancy

node). Therefore, the constraint (6) is violated and the flow should be decompressed before or at least at the destination node $\left(w_{t}=1\right)$.

Although the GreenRE model is already a complex task, it does not take the fluctuation in real-life traffic into account. In practice, the actual traffic demand $D^{s t}$ and the redundant rate $\gamma^{\text {st }}$ fluctuate and are not known in advance. Hence, a Robust-GreenRE model should be proposed to address this issue by taking both traffic demand and redundancy rate uncertainty into account while satisfying the capacity constraints (3).

\subsection{Robust Optimization}

Over the past years, robust optimization has been established as a special branch of mathematical optimization allowing to handle uncertain data $[6,7]$. A specialization of robust optimization, which is particularly attractive by its computational tractability, is the so-called $\Gamma$-robustness concept introduced by Bertsimas and Sim [8,9]. Instead of deterministic coefficients, the coefficients $a_{j}$ of a constraint $\sum_{j} a_{j} x_{j} \leq b$ are assumed to be random variables. Bertsimas and Sim have shown that in case all random variables are independent and have a symmetric distribution of the form $a_{j} \in\left[\bar{a}_{j}-\hat{a}_{j}, \bar{a}_{j}+\hat{a}_{j}\right]$ (with $\bar{a}_{j}$ the average and $\hat{a}_{j}$ the maximum deviation), it can be guaranteed that the constraint is satisfied with high probability by defining an appropriate integer $\Gamma$ and replacing the constraint by

$$
\sum_{j} \bar{a}_{j} x_{j}+\max _{J:|J| \leq \Gamma} \sum_{j \in J} \hat{a}_{j} x_{j} \leq b .
$$

This constraint models that, for each realization of the uncertainties, at most $\Gamma$ many (but arbitrary) coefficients can deviate from their nominal value. Given an arbitrary realization, it is shown in $[8,9]$, that the probability that (9) is violated, is about $1-\Phi\left(\frac{\Gamma-1}{\sqrt{n}}\right)$, where $\Phi$ is the cumulative distribution function of the standard normal distribution and $n$ equals the number of uncertain coefficients. This result is independent of the actual distribution of $a_{j}$.

Note that constraint (9) is deterministic and the complete problem can be reformulated as a standard mixed integer problem. So the model including uncertainty can be solved by the same means as the original problem. Again see $[8,9]$ for details. From a practical perspective, by varying the parameter $\Gamma$, different solutions can be obtained with different levels of robustness (the higher $\Gamma$ is, the more robust, but also the more expensive the solution is). This concept has already been applied to several network optimization problems [30, 1, 19]. 


\section{Robust-GreenRE Model}

Fig. 6 shows real traffic traces of the three source-destination pairs: (a) Washington D.C. Los Angeles, (b) Seattle - Indianapolis, and (c) Seattle - Chicago in the US Abilene Internet2 network in intervals of 5 mins during the first 10 days of July 2004 [30]. We observe that, at some points, each traffic demand can achieve a maximum (peak) value. However, the traffic peaks do not occur simultaneously for the three demands. This confirms an assumption that the number of simultaneous demand peaks is bounded [30]. Hence, we propose in this section a Robust-GreenRE model to deal with this kind of uncertainty.

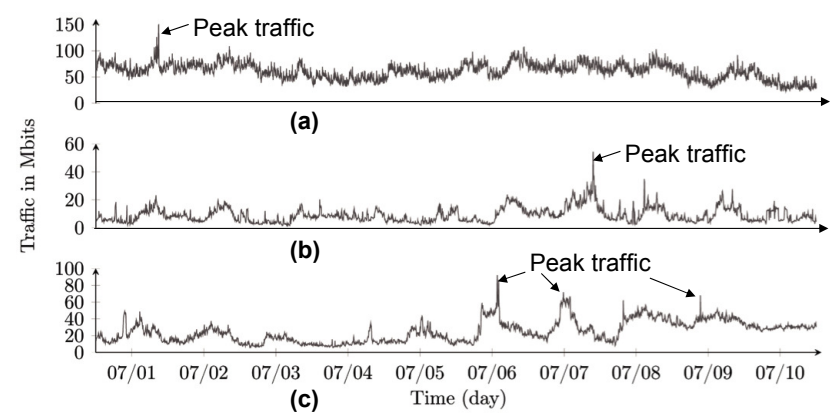

Figure 6: Traffic demands in Abilene network [30]

In the Robust-GreenRE model, two values determining percentage of non-redundant traffic are given for each traffic demand: a nominal (default) value $\bar{\gamma}^{s t} \in(0,1]$ and a deviation $\widehat{\gamma}^{s t}$ such that $0 \leq \widehat{\gamma}^{s t}, \bar{\gamma}^{s t}+\widehat{\gamma}^{s t} \leq 1$ and the actual non-redundant rate $\gamma^{s t} \in\left[\bar{\gamma}^{s t}, \bar{\gamma}^{s t}+\widehat{\gamma}^{s t}\right]$. Similarly, each traffic demand is given by a nominal value $\bar{D}^{s t} \geq 0$ and a deviation $\widehat{D}^{s t} \geq 0$ such that the actual demand volume $D^{s t} \in\left[\bar{D}^{s t}, \bar{D}^{s t}+\widehat{D}^{s t}\right]$. Potentially, each demand is expressed with its default value: $D^{s t}=\bar{D}^{s t}$ and $D_{\text {comp }}^{s t}=\bar{\gamma}^{s t} \times \bar{D}^{s t}$. In the worst case realization, the peak values should be used and each traffic pair is expressed by $D^{s t}=\left(\bar{D}^{s t}+\widehat{D}^{s t}\right)$ and $D_{\text {comp }}^{s t}=\left(\bar{\gamma}^{s t}+\widehat{\gamma}^{s t}\right) \times\left(\bar{D}^{s t}+\widehat{D}^{s t}\right)$. Given two integral parameters $0 \leq \Gamma_{d}, \Gamma_{\gamma} \leq|\mathcal{D}|(|\mathcal{D}|$ is the total number of demands), we denote $Q \subseteq \mathcal{D},|Q| \leq \Gamma_{d}$, a set of traffic pairs allowed to deviate simultaneously from their nominal traffic volumes. Similarly, $Q^{\prime} \subseteq \mathcal{D},\left|Q^{\prime}\right| \leq \Gamma_{\gamma}$, is a set of demands in which all RE rates can deviate simultaneously. Observe that demands in $Q \cap Q^{\prime}$ are simultaneously at their peak traffic and lowest RE rates. Given $\left(\Gamma_{d}, \Gamma_{\gamma}\right)$ as the desired robustness of the network, the RobustGreenRE problem is to minimize the energy consumption of the network while satisfying the link capacity constraints whenever at most $\Gamma_{d}$ demands and $\Gamma_{\gamma} \mathrm{RE}$ rates deviate simultaneously from their nominal values.

Table 1: Demands and redundancy rates variation

\begin{tabular}{|c|c|c|c|c|}
\hline Demand (s, t) & $\bar{D}^{s t}$ & $\widehat{D}^{s t}$ & $\bar{\gamma}^{s t}$ & $\widehat{\gamma}^{s t}$ \\
\hline$(0,3)$ & 3 & 1 & 0.5 & 0.3 \\
\hline$(4,7)$ & 2 & 1 & 0.6 & 0.3 \\
\hline$(8,11)$ & 1 & 2 & 0.7 & 0.3 \\
\hline
\end{tabular}

Let us analyze the example of Fig. 7 to see that it is non-trivial to solve the RobustGreenRE problem. We consider a $(3 \times 4)$ grid with a capacity of $4 \mathrm{Mbps}$ per direction of each links. There are three traffic demands to be routed: $(0,3),(4,7)$ and $(8,11)$, each with respective nominal traffic volumes $\bar{D}^{s t}$ and deviation $\widehat{D}^{s t}$ (resp. nominal RE rates $\bar{\gamma}^{s t}$ and 

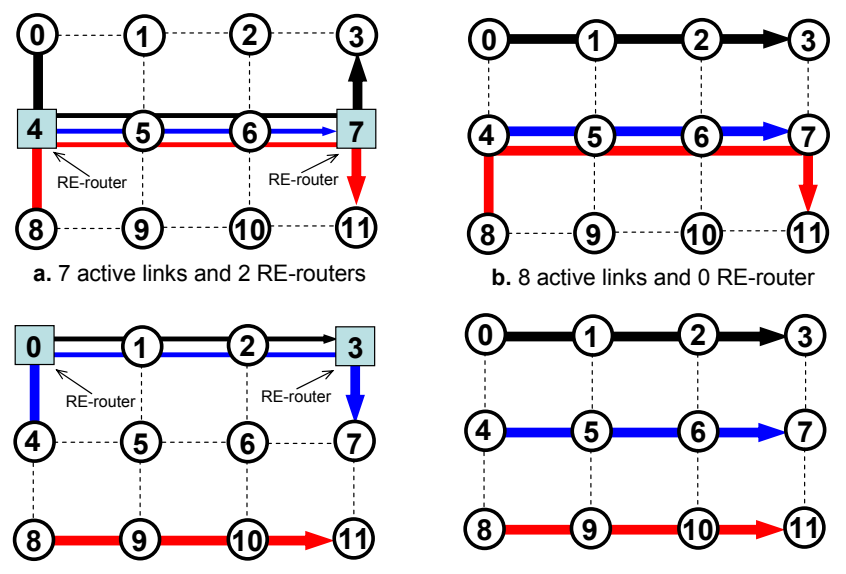

d. 9 active links and 0 RE-router

Figure 7: Example of robustness

deviation $\widehat{\gamma}^{s t}$ ) as shown in Table 1. As shown in Fig. 7a, this is the optimal solution for the case in which no uncertainty is defined $\left(\Gamma_{d}=\Gamma_{\gamma}=0\right)$. In this solution, we activate two RErouters at nodes 4 and 7 and the total traffic passing through links $(4-5-6-7)$ is equal to $\bar{D}^{0,3} \times \bar{\gamma}^{0,3}+\bar{D}^{4,7} \times \bar{\gamma}^{4,7}+\bar{D}^{8,11} \times \bar{\gamma}^{8,11}=3 \times 0.5+2 \times 0.6+1 \times 0.7=3.4<4$.

\begin{tabular}{|c|c|c|c|c|c|c|c|}
\hline Case & $\mathrm{Q}$ & Q' & \multicolumn{2}{|c|}{ Best solution } & \multicolumn{3}{|c|}{ Link load $l_{u v}(\mathrm{Mbps})$} \\
\hline 1 & $(0,3)$ & $(0,3)$ & Fig. 1b & 1600 Watts & $l_{0,1,2,3}=4$ & $l_{4,5,6,7}=3$ & $l_{8,4}=l_{7,11}=1$ \\
\hline 2 & $(0,3)$ & $(4,7)$ & Fig. 1b & 1600 Watts & $l_{0,1,2,3}=4$ & $l_{4,5,6,7}=3$ & $l_{8,4}=l_{7,11}=1$ \\
\hline 3 & $(0,3)$ & $(8,11)$ & Fig. 1b & 1600 Watts & $l_{0,1,2,3}=4$ & $l_{4,5,6,7}=3$ & $l_{8,4}=l_{7,11}=1$ \\
\hline 4 & $(4,7)$ & $(0,3)$ & Fig. 1b & 1600 Watts & $l_{0,1,2,3}=3$ & $l_{4,5,6,7}=4$ & $l_{8,4}=l_{7,11}=1$ \\
\hline 5 & $(4,7)$ & $(4,7)$ & Fig. 1b & 1600 Watts & $l_{0,1,2,3}=3$ & $l_{4,5,6,7}=4$ & $l_{8,4}=l_{7,11}=1$ \\
\hline 6 & $(4,7)$ & $(8,11)$ & Fig. 1b & 1600 Watts & $l_{0,1,2,3}=3$ & $l_{4,5,6,7}=4$ & $l_{8,4}=l_{7,11}=1$ \\
\hline 7 & $(8,11)$ & $(0,3)$ & Fig. 1c & 1660 Watts & $l_{0,1,2,3}=3.6$ & $l_{4,0}=2$ & $l_{8,9,10,11}=3 \quad l_{3,7}=2$ \\
\hline 8 & $(8,11)$ & $(4,7)$ & Fig. 1c & 1660 Watts & $l_{0,1,2,3}=3.3$ & $l_{4,0}=2$ & $l_{8,9,10,11}=3 \quad l_{3,7}=2$ \\
\hline 9 & $(8,11)$ & $(8,11)$ & Fig. 1c & 1660 Watts & $l_{0,1,2,3}=2.7$ & $l_{4,0}=2$ & $l_{8,9,10,11}=3 \quad l_{3,7}=2$ \\
\hline
\end{tabular}

Table 2: 9 cases of the robustness

Consider now the robust case in which $\Gamma_{d}=\Gamma_{\gamma}=1$. There are 9 possible cases for the combinations of deviation in traffic volumes and RE rate as reported in Table 2. In Case 1, demand $(0,3)$ deviates both on its traffic volume and $\mathrm{RE}$ rate. Thus the solution of Fig. 7a is infeasible because the traffic volume passing through links $(4-5-6-7)$ is $\left(\bar{D}^{0,3}+\widehat{D}^{0,3}\right) \times$ $\left(\bar{\gamma}^{0,3}+\widehat{\gamma}^{0,3}\right)+\bar{D}^{4,7} \times \bar{\gamma}^{4,7}+\bar{D}^{8,11} \times \bar{\gamma}^{8,11}=(3+1) \times(0.5+0.3)+2 \times 0.6+1 \times 0.7=5.1>4$. The optimal solution in this case is presented in Fig. $7 \mathrm{~b}$ in which 8 links are activated and no RE-router is used. The power consumption is $8 \times 200=1600$ Watts. In Case 9 , both the traffic volume and the RE rate of demand $(8,11)$ deviate simultaneously. The solution in Fig. $7 \mathrm{~b}$ is infeasible in this case even if we enable RE-routers at node 4 and 7 since the total traffic passing through links $(4-5-6-7)$ will be $\bar{D}^{4,7} \times \bar{\gamma}^{4,7}+\left(\bar{D}^{8,11}+\widehat{D}^{8,11}\right) \times\left(\bar{\gamma}^{8,11}+\widehat{\gamma}^{8,11}\right)=$ $2 \times 0.6+(1+2) \times(0.7+0.3)=4.2>4$. In Case 9, the optimal solution is the one of Fig. $7 \mathrm{c}$ with 8 active links and 2 RE-routers. However, in the Robust-GreenRE model with $\Gamma_{d}=\Gamma_{\gamma}=1$, 
any demand can deviate from its nominal volume or RE rate, as long as at most one demand and one $\mathrm{RE}$ rate deviate their volumes at the same time. Consequently, a solution is feasible if and only if it satisfies all of the 9 cases. Hence, Fig. 7d is the only feasible solution since Fig. 7c is infeasible for Case 1 of Table 2.

The idea of robustness is that we should reserve some space in the link capacity to accommodate the fluctuation in the traffic volumes and RE rates. To do so, we define a function $\delta_{u v}\left(f, g, \Gamma_{d}, \Gamma_{\gamma}\right)$ such that the capacity constraints satisfy:

$$
\sum_{\left(s, t, D^{s t}\right) \in \mathcal{D}} \bar{D}^{s t}\left(f_{u v}^{s t}+\bar{\gamma}^{s t} g_{u v}^{s t}\right)+\delta_{u v}\left(f, g, \Gamma_{d}, \Gamma_{\gamma}\right) \leq \mu C_{u v} x_{\{u, v\}} \quad \forall\{u, v\} \in E
$$

The problem now is to find the value of the function $\delta_{u v}\left(f, g, \Gamma_{d}, \Gamma_{\gamma}\right)$. To answer this question, we use the notations $Q_{d}=Q \backslash Q^{\prime}, Q_{\gamma}=Q^{\prime} \backslash Q$ and $Q_{d \gamma}=Q \cap Q^{\prime}$ as independent sets such that: $Q_{d \gamma}$ contains demands in which both traffic volumes and RE rates can deviate, $Q_{d}$ (resp. $Q_{\gamma}$ ) contains demands in which only traffic volumes (resp. RE rates) can deviate from their nominal values. Indeed, we can formulate the problem using the two sets $Q$ (demands variation) and $Q^{\prime}$ (RE rates variation). However, the final formulation will be non-linear. Therefore the three sets $Q_{d}, Q_{\gamma}$ and $Q_{d \gamma}$ have to be used to overcome this problem. Then the worst case scenario when considering fluctuation on an arc $(u, v)$ is given by:

$$
\begin{aligned}
\sum_{\left(s, t, D^{s t}\right) \in \mathcal{D}} \bar{D}^{s t} f_{u v}^{s t} & +\max _{Q \subseteq \mathcal{D}}\left\{\sum_{\left(s, t, D^{s t}\right) \in Q} \widehat{D}^{s t} f_{u v}^{s t}\right\} \\
& +\sum_{\left(s, t, D^{s t}\right) \in \mathcal{D}} \bar{D}^{s t} \bar{\gamma}^{s t} g_{u v}^{s t}+\max _{Q_{\gamma}=Q^{\prime} \backslash Q}\left\{\sum_{\left(s, t, D^{s t}\right) \in Q_{\gamma}} \bar{D}^{s t} \widehat{\gamma}^{s t} g_{u v}^{s t}\right\} \\
& +\max _{Q_{d \gamma}=Q \cap Q^{\prime}}\left\{\sum_{\left(s, t, D^{s t}\right) \in Q_{d \gamma}}\left(\widehat{D}^{s t} \widehat{\gamma}^{s t}+\widehat{D}^{s t} \bar{\gamma}^{s t}+\bar{D}^{s t} \widehat{\gamma}^{s t}\right) g_{u v}^{s t}\right\} \\
& +\max _{Q_{d}=Q \backslash Q^{\prime}}\left\{\sum_{\left(s, t, D^{s t}\right) \in Q_{d}} \widehat{D}^{s t} \bar{\gamma}^{s t} g_{u v}^{s t}\right\} \leq \mu C_{u v} x_{\{u, v\}} \quad \forall\{u, v\} \in E
\end{aligned}
$$

Obviously, Constraints (3') and (3") are equivalent if $\delta_{u v}\left(f, g, \Gamma_{d}, \Gamma_{\gamma}\right)$ is the maximum part of Constraint (3"). Constraint (3") can be rewritten as a set of many constraints corresponding to all possible sets $Q_{d}, Q_{\gamma}$ and $Q_{d \gamma}$, but the resulting model has an exponential number of constraints. We thus propose three methods to overcome this difficulty. Note that we apply the same technique for the constraints (4) to achieve the robust counterpart. We omit the presentation here as it is similar to (3") where we simply replace the $\operatorname{arc}(u, v)$ with $(v, u)$.

\subsection{Compact formulation}

Given $f_{u v}^{s t}, g_{u v}^{s t}, \Gamma_{d}$, and $\Gamma_{\gamma}$, the function $\delta_{u v}\left(f, g, \Gamma_{d}, \Gamma_{\gamma}\right)$ can be computed by:

$$
\begin{aligned}
\text { (primal) } \quad \delta_{u v}\left(f, g, \Gamma_{d}, \Gamma_{\gamma}\right)=\max \sum_{\left(s, t, D^{s t}\right) \in \mathcal{D}}( & \widehat{D}^{s t} f_{u v}^{s t}\left(z_{u v, Q_{d}}^{s t}+z_{u v, Q_{d \gamma}}^{s t}\right) \\
& +\bar{D}^{s t} \widehat{\gamma}^{s t} g_{u v}^{s t} z_{u v, Q_{\gamma}}^{s t} \\
& +\left(\widehat{D}^{s t} \widehat{\gamma}^{s t}+\widehat{D}^{s t} \bar{\gamma}^{s t}+\bar{D}^{s t} \widehat{\gamma}^{s t}\right) g_{u v}^{s t} z_{u v, Q_{d \gamma}}^{s t} \\
& \left.+\widehat{D}^{s t} \bar{\gamma}^{s t} g_{u v}^{s t} z_{u v, Q_{d}}^{s t}\right)
\end{aligned}
$$




$$
\begin{aligned}
& \text { s.t. } \quad \sum_{\left(s, t, D^{s t}\right) \in \mathcal{D}}\left(z_{u v, Q_{d}}^{s t}+z_{u v, Q_{d \gamma}}^{s t}\right) \leq \Gamma_{d} \\
& \sum_{\left(s, t, D^{s t}\right) \in \mathcal{D}}\left(z_{u v, Q_{\gamma}}^{s t}+z_{u v, Q_{d \gamma}}^{s t}\right) \leq \Gamma_{\gamma} \\
& z_{u v, Q_{d}}^{s t}+z_{u v, Q_{d \gamma}}^{s t}+z_{u v, Q_{\gamma}}^{s t} \leq 1 \\
& z_{u v, Q_{d}}^{s t} \in\{0,1\} \\
& z_{u v, Q_{\gamma}}^{s t} \in\{0,1\} \\
& z_{u v, Q_{d \gamma}}^{s t} \in\{0,1\} \\
& \forall\{u, v\} \in E \quad\left[\pi_{u v, d}\right] \\
& \forall\{u, v\} \in E \quad\left[\pi_{u v, \gamma}\right] \\
& \forall\{u, v\} \in E,\left(s, t, D^{s t}\right) \in \mathcal{D} \quad\left[\sigma_{u v}^{s t}\right] \\
& \forall\{u, v\} \in E \quad\left[\rho_{u v, d}^{s t}\right] \\
& \forall\{u, v\} \in E \quad\left[\rho_{u v, \gamma}^{s t}\right] \\
& \forall\{u, v\} \in E \quad\left[\rho_{u v, d \gamma}^{s t}\right]
\end{aligned}
$$

where binary variables $z_{u v, Q_{d}}^{s t}, z_{u v, Q_{\gamma}}^{s t}$ and $z_{u v, Q_{d \gamma}}^{s t}$ denote whether a traffic pair $\left(s, t, D^{s t}\right)$ belongs respectively to the sets $Q_{d}, Q_{\gamma}, Q_{d \gamma}$ or not. Note that, a traffic demand $\left(s, t, D^{s t}\right)$ belongs exactly to one and only one of the three sets $Q_{d}, Q_{\gamma}$ and $Q_{d \gamma}$. Constraints (3a) and (3b) are used to limit size of the set $|Q|=\left|Q_{d} \cup Q_{d \gamma}\right| \leq \Gamma_{d}$ and $\left|Q^{\prime}\right|=\left|Q_{\gamma} \cup Q_{d \gamma}\right| \leq \Gamma_{\gamma}$. Constraint (3c) indicates that no traffic pair $\left(s, t, D^{s t}\right)$ can belong to more than one of the three sets $Q_{d}, Q_{\gamma}$ and $Q_{d \gamma}$.

We now need to find LP duality of the above primal problem using dual variables $\pi_{u v, d}$, $\pi_{u v, \gamma}, \sigma_{u v}^{s t}, \rho_{u v, d}^{s t}, \rho_{u v, d \gamma}^{s t}$ and $\rho_{u v, \gamma}^{s t}$. To do so, we first relax the last three constraints (3d), (3e) and (3f) to real variables: $0 \leq z_{u v, Q_{d}}^{s t}, z_{u v, Q_{d \gamma}}^{s t}, z_{u v, Q_{\gamma}}^{s t} \leq 1$. By employing LP duality for the relaxation of the primal, we obtain:

$$
\begin{array}{rrr}
\text { (dual) } \quad \delta_{u v}^{r e l a x}\left(f, g, \Gamma_{d}, \Gamma_{\gamma}\right)=\min \Gamma_{d} \pi_{u v, d}+\Gamma_{\gamma} \pi_{u v, \gamma}+\sum_{\left(s, t, D^{s t}\right) \in \mathcal{D}}\left(\sigma_{u v}^{s t}+\rho_{u v, d}^{s t}+\rho_{u v, \gamma}^{s t}+\rho_{u v, d \gamma}^{s t}\right) \\
\text { s.t. } \quad \forall\left(s, t, D^{s t}\right) \in \mathcal{D} \quad \text { (3a') } \\
\pi_{u v, d}+\sigma_{u v}^{s t}+\rho_{u v, d}^{s t} \geq \widehat{D}^{s t}\left(f_{u v}^{s t}+\bar{\gamma}^{s t} g_{u v}^{s t}\right) & \forall\left(s, t, D^{s t}\right) \in \mathcal{D} \quad\left(3 b^{\prime}\right) \\
\pi_{u v, d}+\pi_{u v, \gamma}+\sigma_{u v}^{s t}+\rho_{u v, d \gamma}^{s t} \geq \widehat{D}^{s t} f_{u v}^{s t}+\left(\widehat{D}^{s t} \widehat{\gamma}^{s t}+\widehat{D}^{s t} \bar{\gamma}^{s t}+\bar{D}^{s t} \widehat{\gamma}^{s t}\right) g_{u v}^{s t} & \\
\pi_{u v, \gamma}+\sigma_{u v}^{s t}+\rho_{u v, \gamma}^{s t} \geq \bar{D}^{s t} \widehat{\gamma}^{s t} g_{u v}^{s t} & \forall\left(s, t, D^{s t}\right) \in \mathcal{D} \quad\left(3 c^{\prime}\right) \\
\pi_{u v, d}, \pi_{u v, \gamma}, \sigma_{u v}^{s t}, \rho_{u v, d}^{s t}, \rho_{u v, \gamma}^{s t}, \rho_{u v, d \gamma}^{s t} \geq 0 & \forall\left(s, t, D^{s t}\right) \in \mathcal{D} \quad\left(3 \mathrm{~d}^{\prime}\right)
\end{array}
$$

Since the primal problem is a max problem, the optimal value of the relaxation of the primal $\delta_{u v}^{\text {relax }}\left(f, g, \Gamma_{d}, \Gamma_{\gamma}\right)$ is greater or equal to the original one $\delta_{u v}\left(f, g, \Gamma_{d}, \Gamma_{\gamma}\right)$. As a result, the objective of the duality of the relaxation is also greater or equal to $\delta_{u v}\left(f, g, \Gamma_{d}, \Gamma_{\gamma}\right)$ and it makes the capacity constraint strongly robust. By embedding this duality of the relaxation into (1)-(8), the (strong) Robust-GreenRE problem can be compactly formulated by replacing constraint (3) with:

$$
\begin{aligned}
\sum_{\left(s, t, D^{s t}\right) \in \mathcal{D}}\left(\sigma_{u v}^{s t}+\rho_{u v, d}^{s t}+\rho_{u v, \gamma}^{s t}+\rho_{u v, d \gamma}^{s t}\right)+ & \sum_{\left(s, t, D^{s t}\right) \in \mathcal{D}} \bar{D}^{s t}\left(f_{u v}^{s t}+\bar{\gamma}^{s t} g_{u v}^{s t}\right) \\
& +\Gamma_{d} \pi_{u v, d}+\Gamma_{\gamma} \pi_{u v, \gamma} \leq \mu C_{u v} x_{\{u, v\}} \quad \forall\{u, v\} \in E
\end{aligned}
$$

and adding constraints (3a'), (3b'), (3c') and (3d') to the deterministic model (1)-(8). Similarly, we do the same way to create and add constraints (4a'), (4b'), (4c') and (4d') for the arc $(v, u)$ to replace the constraint (4) in the deterministic model. 


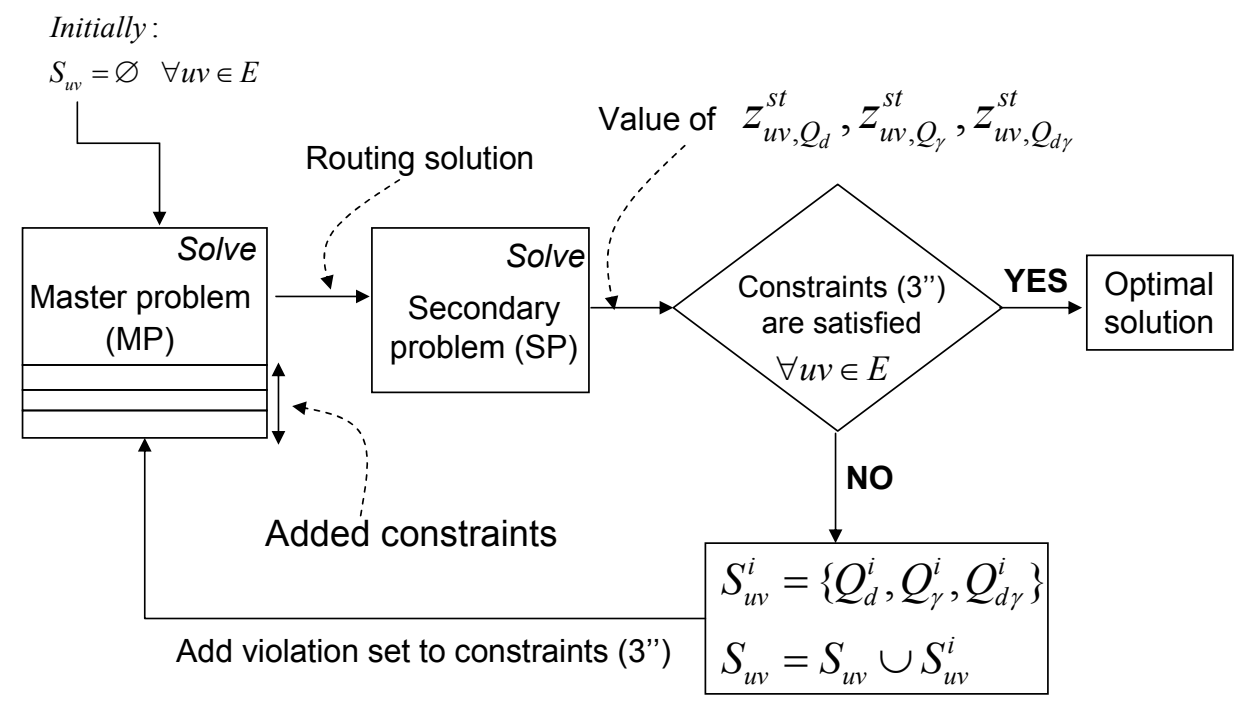

Figure 8: Diagram of constraint generation method

\subsection{Constraint generation (Exact Algorithm)}

As there is a duality gap between the primal and dual models due to the integrality of the primal model, we cannot simply get the optimal solution using the compact formulation based on Bertsimas and Sim methodology like in existing works [30, 18]. In other words, the result obtained from the compact formulation is a lower bound on energy saving. In this section, we present an algorithm that aims at finding the exact solution of the Robust-GreenRE model that includes the objective (1), the Constraints (2), (5) to (8) and the Constraints (3") and (4"). It turns out however that this robust model contains an exponential number of constraints corresponding to Constraint (3") due to the large number of demand sets. To avoid generating all these constraints, we propose in this section a constraint generation algorithm. The main idea of this algorithm is to generate iteratively subsets of traffic demands representing demands which traffic volumes and/or RE rates may deviate from their nominal values and that violate the current solution. Let us call:

- Master Problem (MP): deterministic ILP formulated with Constraints (1)-(8);

- Secondary Problem (SP): primal model of the compact formulation, so Constraints (3a)(3f) with the primal objective function.

We define for each $\operatorname{arc}(u, v)$ of the network a set $S_{u v}^{i}=\left\{Q_{d}^{i}, Q_{d \gamma}^{i}, Q_{\gamma}^{i}\right\}$ of demands which does not satisfy the constraints (3") (or (3')) at iteration $i$ of the algorithm and $S_{u v}=\left\{S_{u v}^{i}\right\}$ (Fig. 8).

Initially, we set $S_{u v}=\emptyset$ and $S_{v u}=\emptyset$ for all $\{u, v\} \in E$. We start the algorithm by solving the MP to find a first feasible routing. Then, we use the values of $f_{u v}^{s t}$ and $g_{u v}^{s t}$ given by the routing solution as inputs for determining $\delta_{u v}\left(f, g, \Gamma_{d}, \Gamma_{\gamma}\right)$ using the SP. Based on the objective value of the SP, we check if constraints (3") are satisfied or not for each arc. As soon as we find a capacity violation on an arc, we use the values of $z_{u v, Q_{d}}^{s t}, z_{u v, Q_{d \gamma}}^{s t}$ and $z_{u v, Q_{\gamma}}^{s t}$ to determine $Q_{d}^{i}, Q_{d \gamma}^{i}, Q_{\gamma}^{i}$. We define $S_{u v}^{i}$ and update $S_{u v}=S_{u v} \cup S_{u v}^{i}$. Finally, we add a new constraint corresponding to the violated constraint (3") and $S_{u v}^{i}$ to the Master Problem. This new constraint prevents the demands in $S_{u v}^{i}$ to be routed simultaneously on the same link. This process is repeated until there is no more violation. If at one step, the Master Problem is infeasible, we conclude 
that there is no solution satisfying the robustness. Otherwise, the final solution is optimal for Robust-GreenRE.

It is important to understand that the feasible solutions (resp. upper bounds) of the MP found in the intermediate iterations are not feasible solutions (resp. upper bounds) for RobustGreenRE. Indeed, the solution computed by MP at intermediate iteration is feasible only for some subsets of $\Gamma_{d}$ and $\Gamma_{\gamma}$ demands deviating but not all of them. Therefore, it can not represent the value $\delta_{u v}\left(f, g, \Gamma_{d}, \Gamma_{\gamma}\right)$ as expressed in the primal model. This is more explained in the following example. Nonetheless, the lower bounds of the intermediate steps are valid lower bounds for Robust-GreenRE.

Let us consider a network with nodes $V=\{A, B, C\}$ and bi-directional links $E=\{A B, A C, B C\}$. Each link has a capacity of $2 \mathrm{Mbps}$ in each direction. Let us also consider the set of demands $\mathcal{D}=\{(A, B),(A, C),(B, C)\}$ all with nominal volume of $1 \mathrm{Mbps}$, deviation volume of $0.5 \mathrm{Mbps}$ and no compression rate. We set $\Gamma_{d}=33 \%$ and $\Gamma_{\gamma}=0 \%$.

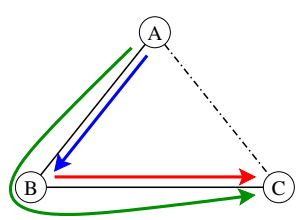

(a) Link $A C$ in sleep mode. New violations on $A B$ and $B C$

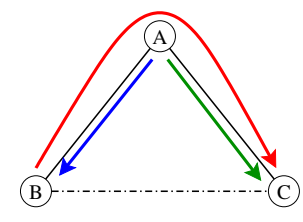

(b) Link $B C$ in sleep mode. New violation on $A C$

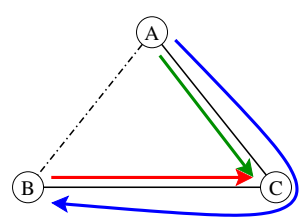

(c) Link $A B$ in sleep mode. New violation on $A C$

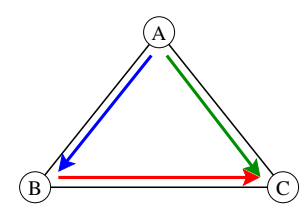

(d) No violation.

Figure 9: Example of iterations of the CG method

Fig. 9 shows different possible iterations of the CG method (the sequence is not unique). The first solution, reported in Fig 9a, is a feasible routing computed by MP when no deviation is allowed. Since $\Gamma_{d}=33 \%$, at least one demand can deviate its volume and we identify with SP capacity violations on links $A B$ and $B C$. Therefore, we add constraints for sets $S_{A B}^{1}=$ $\{(A, B),(A, C)\}$ and $S_{B C}^{1}=\{(B, C),(A, C)\}$ to MP and then proceed with next iteration. The CG method reports successively the solution of the Fig. 9b with capacity violation on link $A C$, the solution of the Fig. 9c with capacity violation on link $A C$, and the solution of Fig. 9d without capacity violation. Consequently, the solution of Fig 9d is optimal for this instance. We observe that the optimal solution has 3 active links, while the solutions found at intermediate iterations have only 2 active links, and so a lower power consumption than the optimal solution for Robust-GreenRE. This shows that the cost of the feasible solutions (and so upper bounds) reported at each intermediate iteration of the CG method are not valid upper bounds for Robust-GreenRE. Recall however that the lower bounds computed at intermediate iterations are valid lower bounds for Robust-GreenRE.

\subsection{Heuristic Algorithm}

Energy-aware routing problem is known to be NP-Hard [22]. Also we now present a heuristic algorithm based on the compact ILP formulation to quickly find efficient solutions for large networks. Since the power consumption of a link (200 Watts [16]) is much more than an enabled RE-router (30 Watts [23]), the heuristic gives priority to minimize of the number of active links. In summary, the heuristic algorithm has two steps: the first step is to use as few active links as possible, and then we minimize the number of RE-routers in the second step.

Step 1 of Algorithm 1 is a constraints satisfaction problem returning a feasible routing. Hence, we use the MILP of the compact formulation without objective function. Our simulations 


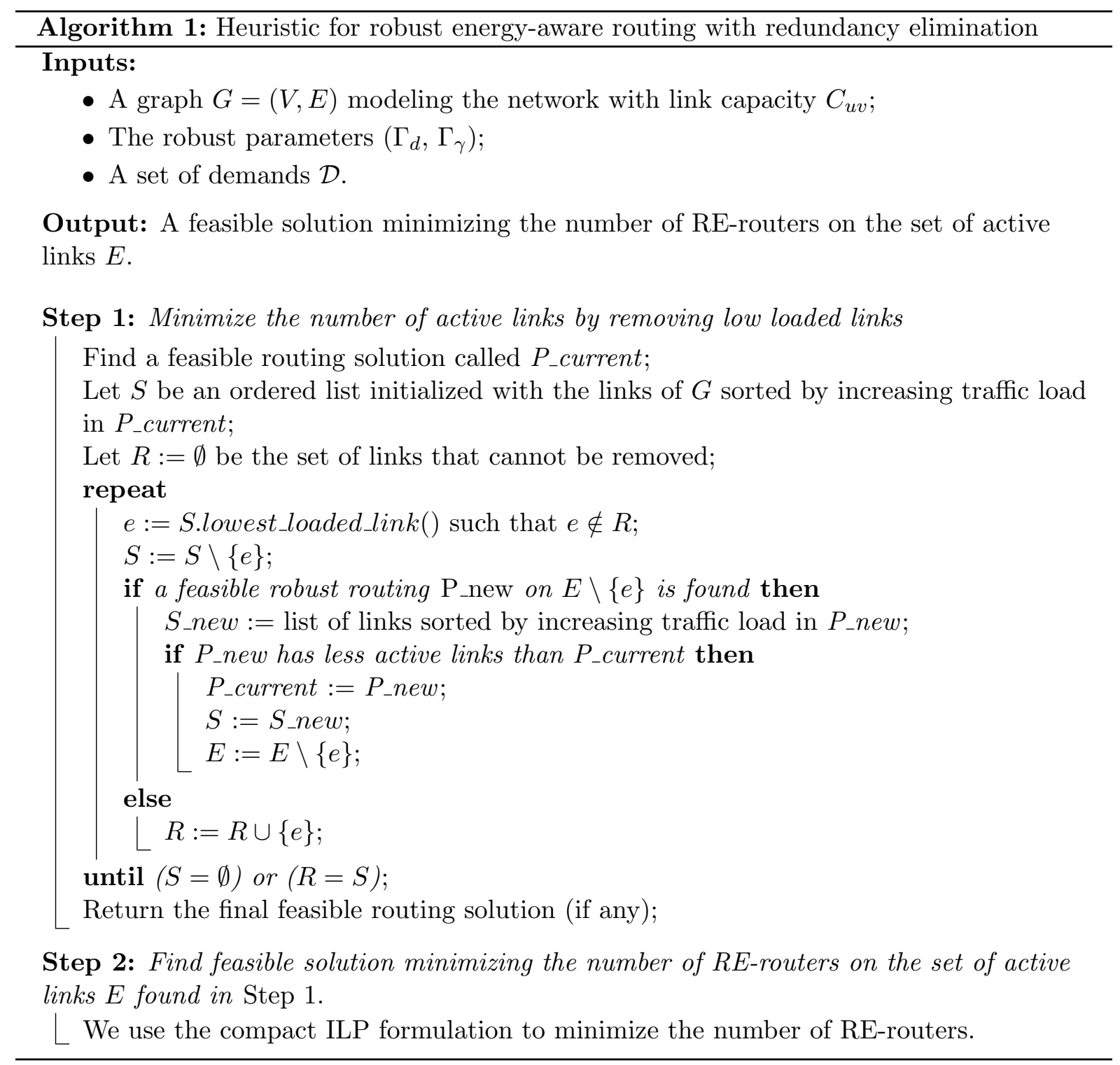


show that it is quite fast to find such a feasible routing solution even for large networks (see Section 4). In each round of the algorithm, we try to remove a link with low load and then to find and evaluate a new feasible routing using less active links. The idea behind this algorithm is that we try to turn off low loaded links and to accommodate their traffic on other links in order to reduce the total number of active links. Observing that unused links (i.e. links that are not carrying traffic) are not considered in the set $S$ since the removal of such a link will result in a routing $P_{-}$new equal to the routing $P_{-}$current.

If a feasible routing is found in Step 1, and so a set of active links, we proceed in Step 2 to minimize the number of enabled RE-routers. More precisely, we use the compact ILP formulation in which the objective function is set to $\min \sum_{u \in V} w_{u}$. Furthermore, we set all binary variables associated to active links to 1 and the others to 0 (this speed-up the resolution of the MILP).

To further reduce the computation time of Algorithm 1, we can consider additional heuristic. For instance, in Step 1, while removing a low loaded link (and so setting a binary variable to 0) we can also set the variable $x_{\{u, v\}}$ associated to a heavily loaded link to 1 . Indeed, such link will certainly be part of the final solution. In addition, we can add some valid cut-inequalities to speed-up the resolution of the MILP [31].

\section{Computational Evaluation}

\subsection{Test instances and Experimental settings}

We solved the Robust-GreenRE model with IBM ILOG CPLEX 12.4 solver [29]. All computations were carried out on a computer equipped with a $2.7 \mathrm{Ghz}$ CPU and 8 GB RAM. We consider real-life traffic traces collected from the SNDlib [35]: the U.S. Internet2 Network (Abilene) $(|V|=12,|E|=15,|\mathcal{D}|=130)$, the Geant network $(|V|=22,|E|=36,|\mathcal{D}|=387)$ and the Germany50 $(|V|=50,|E|=88,|\mathcal{D}|=1595)$. Note that, in section 4.2.1, we use a simplified Abilene network in which only a half of demands are used (65 demands, randomly chosen). It is because an exponential number of constraints can be added to the constraint generation model and so the overall computation time is more than 10 hours. Capacity is set to $C_{u v}=5 / 10 / 20$ Gbps for each arc of the Abilene/ Germany50/ Geant network, respectively.

In our test instances, each traffic demand has two values: the nominal and the peak volumes during one day period. These values can be collected using the dynamic traffic from the SNDlib. To achieve a network with high link utilization, all traffic was scaled with a factor of three. To avoid individual bottlenecks, we add parallel links to increase capacity on some specific links. To find parallel links, we first relax the variables $x_{\{u, v\}}$ to integer variables in the Master Problem. Then, we find the routing solution for the worst case scenario $\left(\Gamma_{d}=\Gamma_{\gamma}=100 \%\right)$ using the relaxed Master Problem. The links $\{u, v\}$ in which $x_{\{u, v\}}>1$ would be the congested links, so we add more capacity to these links and call them parallel links. According to [2,3], based on real traffic traces, an upper bound on traffic redundancy is assumed to $50 \%$. In the simulations, we use $\bar{\gamma}=0.5$ and $\widehat{\gamma}=0.3$ and for each scenario, we vary the robust parameters $\left(\Gamma_{d}, \Gamma_{\gamma}\right)$ in between 0 and the total demands $(|\mathcal{D}|)$.

\subsection{Results and Discussion}

Before discussing particular trends or characteristics of solutions, we want to give a visualization of a typical solution of Robust-GreenRE. In Fig. 10, we present solutions for the Abilene network. The figure indicates by line thickness, that the edge is employed with parallel links. In the initial network, there are 4 parallel links as in the last subfigure in Fig. 10. When reducing values of $\Gamma_{d}$ and $\Gamma_{\gamma}$, less capacity is need. Therefore, we can turn off some of the parallel links. However we 


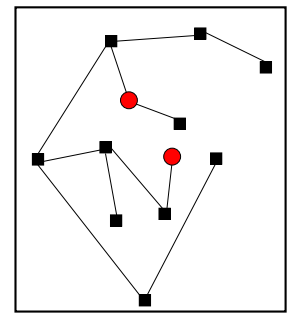

$\Gamma_{\mathrm{y}}=\Gamma_{\mathrm{d}}=0$

\# RE-routers $=2 ;$ \# links $=11$

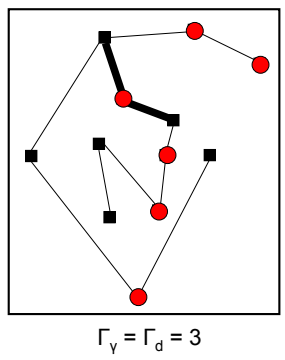

\# RE-routers $=6$; \# links $=13$
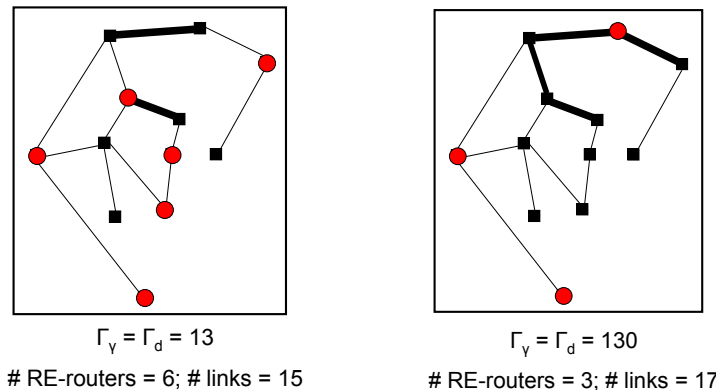

Figure 10: Routing and RE-router placement on Abilene network

still keep one link to connect between two routers and these parallel links become single link. It is noted, that the $\Gamma_{\gamma}=\Gamma_{d}=0$ case mirrors the GreenRE model with nominal demands and RE rates while $\Gamma_{\gamma}=\Gamma_{d}=130$ means the GreenRE model is with all peak values of traffic demands and $\mathrm{RE}$ rates. The subset of chosen edges is printed black and the activated RE-routers are displayed as circles. In a typical solution, between two and six RE-routers are activated. We observed that this number can change independently of the $\Gamma$ value. For instance, 2 RE-routers are needed when $\Gamma_{\gamma}=\Gamma_{d}=0$. This number increases to 6 when $\Gamma_{\gamma}=\Gamma_{d}=3$ or 13. However, the number of RE-routers reduces to 3 when $\Gamma_{\gamma}=\Gamma_{d}=130$. A prognosis is difficult to give, since the number of RE-routers is highly dependent on the traffic volumes, the capacity, and the network topology. Clearly, the same holds for the employed edges and depending on the demands and the employed RE-routers. However, in general, an increase in $\Gamma$ leads to higher capacity requirement and more links and/or RE-routers need to be used.

\subsubsection{Gap between different methods}

In this section, we compare on the simplified Abilene network the energy saving offered by the three proposed methods: Constraints Generation (CG), Compact Formulation (CF), and Heuristic.

We have reported in Table 3 for various values of $\Gamma_{\gamma}, \Gamma_{d}$ the optimality gap of the solutions obtained with each method in less than 10 hours of computation. For CG method, we have also reported the number of violations which corresponds to the number of added constraints. We observe that an increase in the level of robustness (represented by $\Gamma_{\gamma}$, and $\Gamma_{d}$ ) leads to a higher number of violations and so to a larger computation time. The CG method can find optimal solution in less than 10 hours for small $\Gamma_{\gamma}, \Gamma_{d}$ and for $\Gamma_{\gamma}=\Gamma_{d}=100 \%$ (i.e., all traffic demands are at their peaks). However, when $\Gamma_{\gamma}=\Gamma_{d}=10 \%$ and $20 \%$, the CG method is not able to find optimal solutions. As explained in Section 3.2, as long as the optimality of the solution is not proven (i.e. capacity violation is found), we have no guarantee that the returned solution is a feasible solution for Robust-GreenRE. Nonetheless, since we get optimal solution for the case $\Gamma_{\gamma}=\Gamma_{d}=100 \%$ which is the worse case scenario, we can use this solution to evaluate the lower bounds of the CG method when $\Gamma_{\gamma}=\Gamma_{d}=10 \%$ and $20 \%$. This way, we conclude that the optimality gaps for these cases are around $20 \%$.

The CF method is much faster for these instances since it is able to prove the optimality of the solution before the time limit in all cases but when $\Gamma_{\gamma}=\Gamma_{d}=10 \%$ of total demands. In this later case, a solution with optimality gap of $2.5 \%$ is returned. Recall nonetheless that CF computes only a lower bound on the possible energy-saving offered by Robust-GreenRE.

Finally, and as expected, the heuristic algorithm is the fastest method. All feasible solutions are found in less than 50 seconds. To evaluate the quality of the solutions returned by the 
heuristic, we have reported in Table 3 the optimality gap as the ratio of the value of the solution computed with the heuristic over the best lower bound returned by the solver for the CG method. The optimality gap is less than $5 \%$ for small values of $\Gamma_{\gamma}, \Gamma_{d}$, and it is around $25 \%$ for larger values. Note that these gaps are close to the ones found with the CG method. We recall that the CG method should output the optimal solution of the Robust-GreenRE model, however it takes very long execution time. This indicates that the heuristic is able to quickly return good solutions.

\begin{tabular}{|c|r|c|r|r|r|r|c|}
\hline \multirow{2}{*}{$\Gamma_{\gamma}, \Gamma_{d}(\%)$} & \multicolumn{3}{|c|}{ CG method } & \multicolumn{2}{c|}{ CF method } & \multicolumn{2}{c|}{ Heuristic } \\
\cline { 2 - 8 } & \# violations & gap opt (\%) & time (s) & gap opt (\%) & time (s) & gap opt (\%) & time (s) \\
\hline 0 & 0 & 0 & 700 & 0 & 150 & 0 & $\leq 50$ \\
\hline 2 & 5870 & 0 & 1800 & 0 & 1240 & 4 & $\leq 50$ \\
\hline 5 & 36164 & 0 & 23300 & 0 & 9000 & 5 & $\leq 50$ \\
\hline 10 & 64841 & 18.9 & 36000 & 2.5 & 36000 & 24 & $\leq 50$ \\
\hline 20 & 64433 & 20.6 & 36000 & 0 & 22000 & 27 & $\leq 50$ \\
\hline 100 & 65576 & 0 & 36000 & 0 & 1400 & 7 & $\leq 50$ \\
\hline
\end{tabular}

Table 3: Constraint Generation (CG) vs. Compact Formulation (CF) vs. Heuristic for Abilene network.

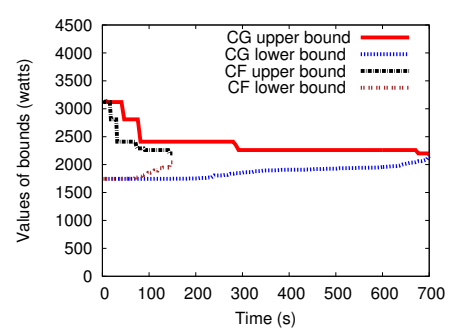

(a) Abilene $\Gamma_{d}=\Gamma_{\gamma}=0 \%$

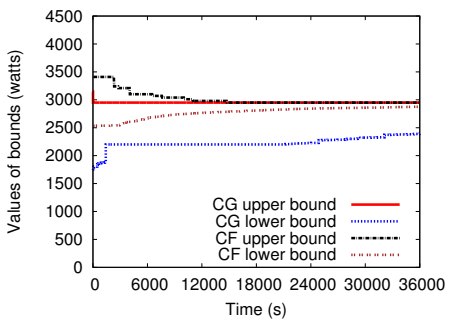

(d) Abilene $\Gamma_{d}=\Gamma_{\gamma}=10 \%$

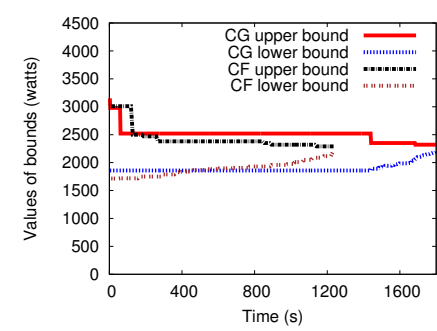

(b) Abilene $\Gamma_{d}=\Gamma_{\gamma}=2 \%$

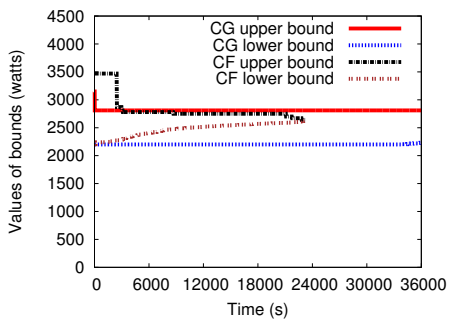

(e) Abilene $\Gamma_{d}=\Gamma_{\gamma}=20 \%$

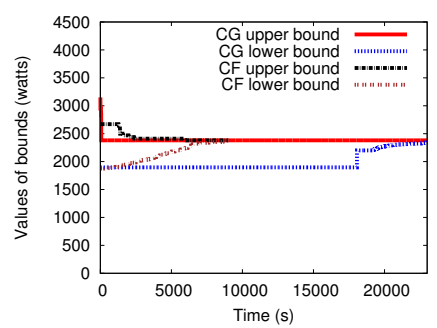

(c) Abilene $\Gamma_{d}=\Gamma_{\gamma}=5 \%$

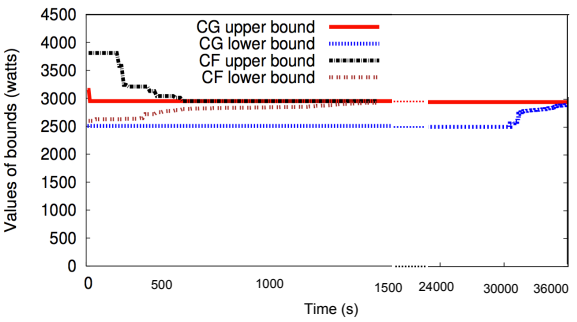

(f) Abilene $\Gamma_{d}=\Gamma_{\gamma}=100 \%$

Figure 11: Upper bound and lower bound: Compact Formulation (CF) vs. Constraint Generation $(C G)$

To perform a deeper analysis of behavior of the Constraints Generation (CG) and Compact Formulation $(\mathrm{CF})$ methods, we have plotted in Figs. 11 and 12 the evolution over time of respectively the upper bound, the lower bound (Fig. 11), and the optimality gap (Fig. 12) obtained by the solver. Recall that the upper bounds reported for CG method before the optimality proof are not necessarily the upper bounds for Robust-GreenRE, but the lower bounds are. We observe that the main drawback of the CG method for these instances is in the lower bounds. These bounds are very low and the CG method needs a lot of time to improve them and so reduce the optimality gap. For instance, in the case $\Gamma_{d}=\Gamma_{\gamma}=20 \%$ reported in Fig. 11e, the improvement of the initial lower bound after 10 hours of computation is almost 


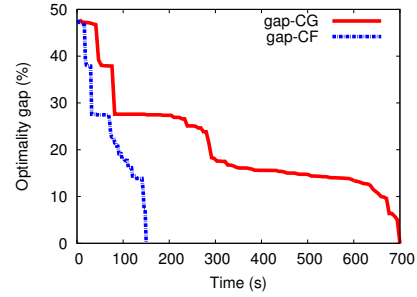

(a) Abilene $\Gamma_{d}=\Gamma_{\gamma}=0 \%$

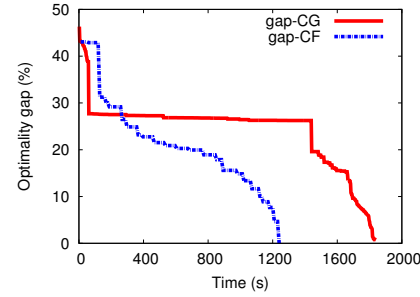

(b) Abilene $\Gamma_{d}=\Gamma_{\gamma}=2 \%$

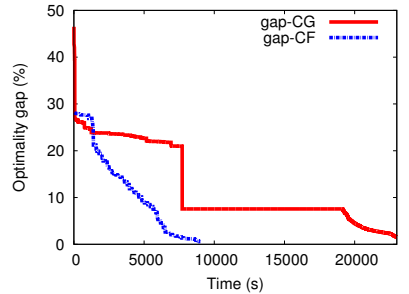

(c) Abilene $\Gamma_{d}=\Gamma_{\gamma}=5 \%$

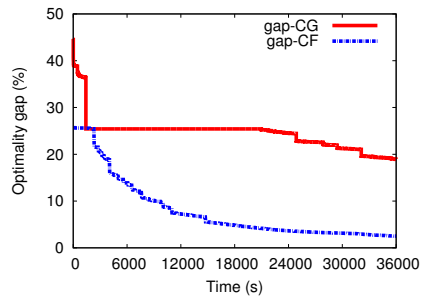

(d) Abilene $\Gamma_{d}=\Gamma_{\gamma}=10 \%$

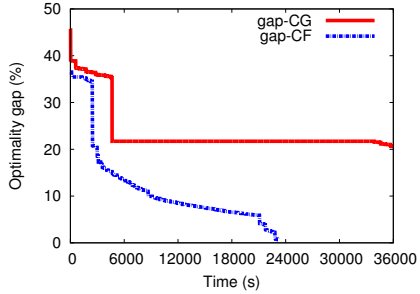

(e) Abilene $\Gamma_{d}=\Gamma_{\gamma}=20 \%$

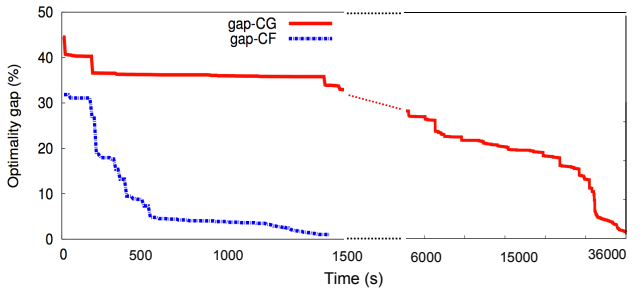

(f) Abilene $\Gamma_{d}=\Gamma_{\gamma}=100 \%$

Figure 12: Optimality gaps: Compact Formulation (CF) vs. Constraint Generation (CG)

null. The CF method has a different behavior since both upper and lower bounds are regularly improved, and except for the case $\Gamma_{d}=\Gamma_{\gamma}=10 \%$, the optimality of the current feasible solution is improved much before the time limit of 10 hours. Furthermore, the upper bounds of the CF method correspond to feasible solutions for Robust-GreenRE. In general, the CG method can quickly achieve a good upper bound while the CF method is good in finding the lower bound.

We show in Fig. 12 another view of the evolution: the evolution of the optimality gap. When this gap reaches zero, the optimality of the current feasible solution is proved. Clearly, the CF method outperforms the CG method in term of improving optimality gap for these instances. However, it is noted that we can only find the exact solution using the CG method. The optimal solution obtained with the CF method is only a lower bound on the energy saving that can be made with the CG method (see section 3.2).

We now compare in Fig. 13 the performances of the three methods in terms of energy saving (y-axis) for various levels of robustness (x-axis). In this plot, both $\Gamma_{d}$ and $\Gamma_{\gamma}$ vary with the same value, e.g. robustness $=5 \%$ means $\Gamma_{d}=\Gamma_{\gamma}=0.05 \times|\mathcal{D}|$. The percentage of energy saving is the ratio of the amount of energy saved in the Robust-GreenRE case over the total amount of energy consumed when all links are activated. It is computed using the following formula: $\frac{\left(200\left|E^{\prime}\right|-30 W\right)}{200|E|}$ where $\left|E^{\prime}\right|$ is the number of links in sleep mode, $W$ is the number of RE-routers, and $|E|$ is the total number of links in the network (see the example in Section 2.3.1). We have not reported energy saving for the CG method with $\Gamma_{d}=\Gamma_{\gamma}=10 \%$ and $20 \%$ since we were not able to find feasible solution for Robust-GreenRE in these cases.

We observe that the maximum gap reported in Fig. 13 between the heuristic and the CG (and $\mathrm{CF}$ ) method is $7.63 \%$, and this gap decreases for small values of $\Gamma_{d}$ and $\Gamma_{\gamma}$. Recall that measurements performed on real networks have shown that only a small fraction of the traffic demands deviate simultaneously from their nominal values [30]. Furthermore, the aim of robust optimization is precisely to take benefit of that fact in order to improve the design of the network, and in our case to save more energy. We have seen that our heuristic algorithm offers good performances both in terms of running time and quality of the solution in this setting. Thus in the sequel, we will use our heuristic to evaluate the Robust-GreenRE model on larger instances. 


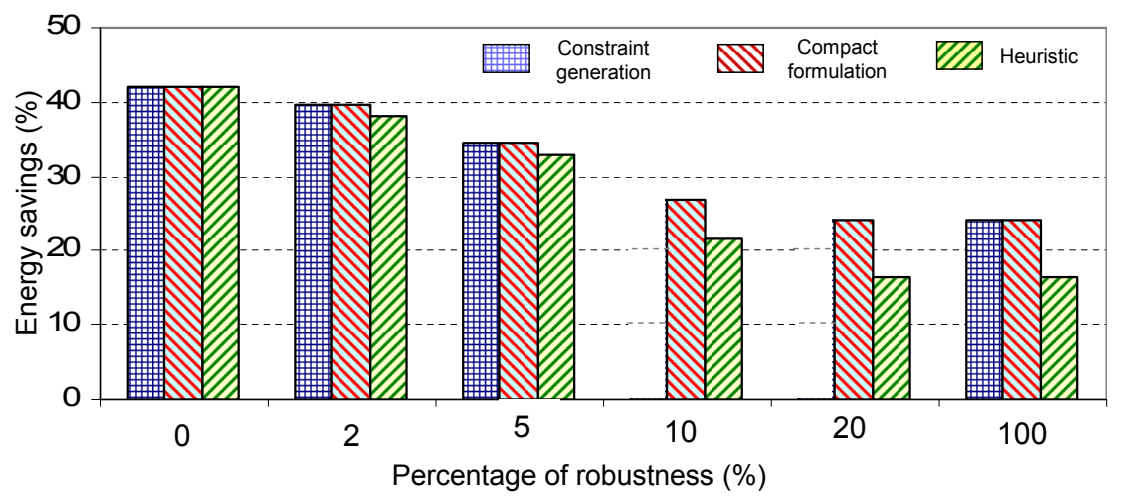

Figure 13: Comparison of proposed methods on Abilene.

Finally, we have to mention that the CG method was not able to find any feasible solution for Geant and Germany50 networks within the 10 hour computation time limit. Furthermore, the feasible solutions found (if any) with the CF method for these networks were worse than the solutions computed by the heuristic. Indeed, the CF method involves such a large number of variables and constraints for these instances, that we can hardly expect to find good feasible solutions within acceptable computation time. The CG and CF methods can thus be used only for instances at the scale of the Abilene instance, and they were useful to evaluate the behavior of the heuristic on such instances.

\subsubsection{Energy saving vs. robustness}

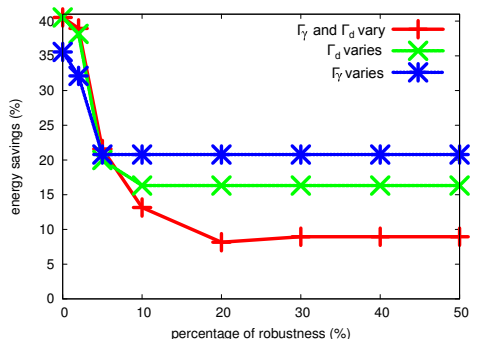

(a) Abilene

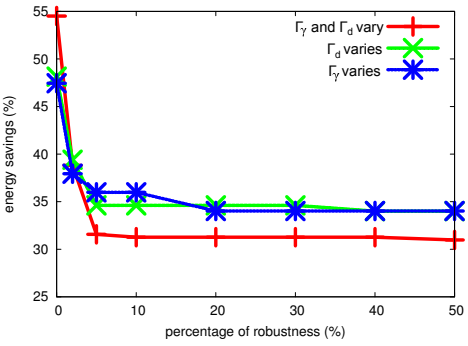

(b) Geant

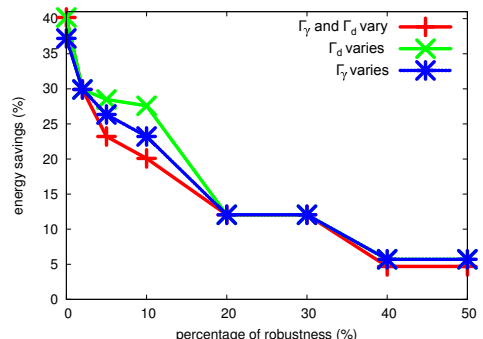

(c) Germany50

Figure 14: Energy saving vs. robustness for Abilene, Geant and Germany50 network

Fig. 14 shows the trade-off between energy saving and the level of robustness regarding the parameters $\left(\Gamma_{d}, \Gamma_{\gamma}\right)$. We consider three test cases (1) both $\Gamma_{d}$ and $\Gamma_{\gamma},(2)$ only $\Gamma_{\gamma}$ and (3) only $\Gamma_{d}$ vary their values. In the Case 1 , both $\Gamma_{d}$ and $\Gamma_{\gamma}$ vary with the same value of robustness. Note that, when $\Gamma_{\gamma}=\Gamma_{d}=100 \%$, all demands and compression rates are at the worst case, therefore the Robust-GreenRE is equivalent to the deterministic GreenRE. In Case 2 (resp. Case 3 ), while $\Gamma_{\gamma}\left(\right.$ resp. $\left.\Gamma_{d}\right)$ varies, $\Gamma_{d}$ (resp. $\Gamma_{\gamma}$ ) is set to $2 \%$ of the total demands. In all the three networks, the solutions do not change when $\Gamma_{d}, \Gamma_{\gamma} \geq \frac{|\mathcal{D}|}{2}$, thus the x-axis is cut at $50 \%$. We observe that energy saving is proportional to $1 / \Gamma$. Indeed, large values of $\Gamma$ reduce the interest for robust optimization. More precisely, when $\Gamma_{d}, \Gamma_{\gamma} \geq 30 \%$, energy saving offered by the Robust-GreenRE model is almost the same as the GreenRE model, while when $\Gamma_{d}, \Gamma_{\gamma} \leq 20 \%$ the Robust-GreenRE model allows for significant energy saving. An explanation of this phenomenon can be found in the distribution of the demand volumes. A small fraction 
of the demands dominates the others in volume. Hence, when the values of $\Gamma_{d}, \Gamma_{\gamma}$ covers all of these dominating demands, increasing $\Gamma_{d}, \Gamma_{\gamma}$ does not affect the routing solution and the percentage of energy saving remains stable. In Case 2 and Case 3 , when only $\Gamma_{d}$ or $\Gamma_{\gamma}$ varies its value, the same phenomenon is observed. It means $\Gamma_{d}$ and $\Gamma_{\gamma}$ have almost the same role in contributing to the robustness of the network.

\subsubsection{Link utilization}

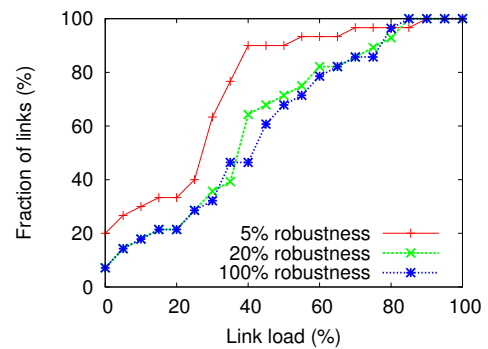

(a) Abilene

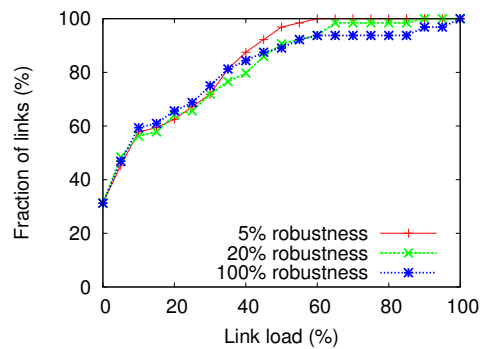

(b) Geant

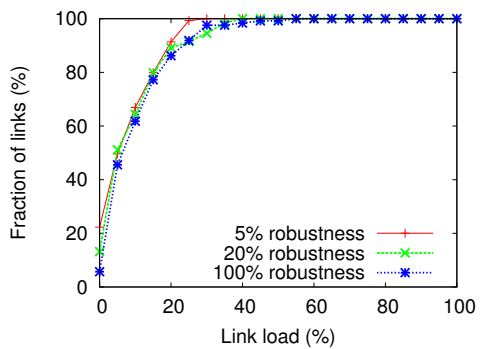

(c) Germany50

Figure 15: CDF load of all links including links in sleep mode for Abilene, Geant and Germany50 networks.

We now evaluate the impact of Robust-GreenRE on links utilization. Intuitively, since any energy-aware routing scheme operating in the Idle Energy model, as described in Section 2.1, aims at minimizing the number of active links, fewer links are used to carry the traffic. Consequently, active links are expected to be highly loaded. To analyze this behavior, we have plotted in Fig. 15 the cumulative distribution function (CDF) of the links load for Abilene, Geant and Germany50 networks. The CDF describes the fraction of the links having their utilizations (loads) less or equal to a given value. Here, the utilization of a link is measured as the maximum load induced by the routing for any subset of $\Gamma_{d}$ demands that are simultaneously at their peak and any subset of $\Gamma_{\gamma}$ with reduced redundancy elimination rate. More precisely, the utilization of a link is computed as the value of the left hand side of the constraint ( 3 '). Thus, this is the worst case scenario in the range of the allowed fluctuation defined by $\Gamma_{d}$ and $\Gamma_{\gamma}$.

In Fig. 15 we include links that are in sleep mode and so with null load. For ease of observation, we only show three cases of robustness for each network, the other cases follow similar curve patterns. As shown in Fig. 15, Geant and Germany50 networks have low traffic load. For instance, $80 \%$ of the links of Geant and Germany50 networks have a load respectively under $40 \%$ and $20 \%$ of their capacities. Traffic on Abilene network is heavier, however there is no overloaded link and $80 \%$ of the links have an utilization of less than $70 \%$. Since a higher value of robustness means that more traffic demands are at peak values, the computed link utilization is high when the level of robustness is high. For example, in Abilene network with $5 \%$ of robustness, $85 \%$ of the links are under $40 \%$ utilization, while for $20 \%$ (resp. 100\%) of robustness, it is only $65 \%$ (resp. $45 \%$ ) of the links that are under $40 \%$ utilization.

\subsubsection{Robust-GreenRE vs. GreenRE vs. Classical EAR}

In Fig. 16, we compare the Robust-GreenRE model with the GreenRE and the classical EAR (no compression) models for small values of $\Gamma_{d}$ and $\Gamma_{\gamma}$. Since the GreenRE model does not take into account RE rate deviation, we set $\gamma^{s t}=0.8$ (20\% of traffic is redundant) and for EAR model , $\gamma^{\text {st }}$ is set to 1.0 (no compression). Furthermore, since traffic volume variations are not 


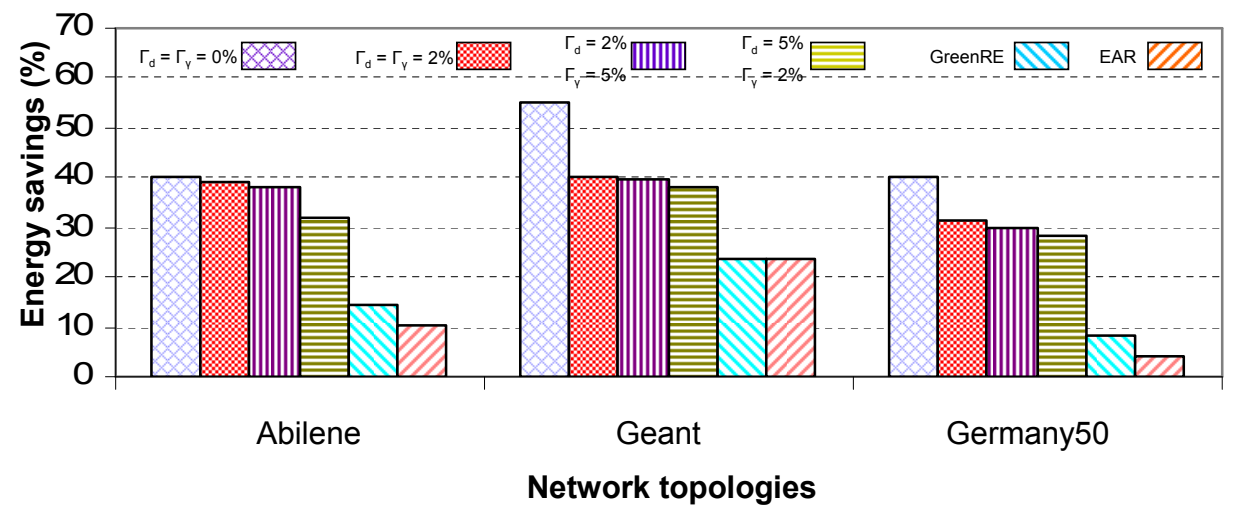

Figure 16: Robust-GreenRE vs. GreenRE vs. EAR.

handled by GreenRE and EAR models, all demands are at peak. When $\Gamma_{d}=\Gamma_{\gamma}=0 \%$, all traffic demands are at their nominal values, the Robust-GreenRE model becomes the GreenRE model with nominal traffic demands, namely the GreenRE $E_{n o m i n a l}$. Therefore, energy saving of the Robust-GreenRE model is in between that of the GreenRE $E_{\text {nominal }}$ and the GreenRE models. We observe that, in Germany50 network, the EAR and the GreenRE models offer a small amount of energy saving. A forecast is difficult to give, since energy saving is dependent on both the network topology and the traffic matrix. One parameter that can be used to explain the phenomenon is that the volume of peak traffic in Germany50 network is much bigger than the nominal one (the average ratio of the peak over the nominal traffic is around 6). That is why the Robust-GreenRE model provides higher energy saving than the EAR and the GreenRE model in Germany50 network. It is noted that the Robust-GreenRE model is more efficient than the GreenRE model when only few traffic demands fluctuate their volumes and RE rates ( $\Gamma$ is relatively small). When $\Gamma$ is quite big, e.g. $\Gamma>=20 \%$, the Robust-GreenRE and the GreenRE models yield almost the same amount of energy saving (as shown in Fig. 14). However, this result does not invalidate the benefit of $\Gamma$-robustness because in real-life traffic, only a few demands will vary their traffic simultaneously [30]. In summary, when $\Gamma=2-5 \%$, the Robust-GreenRE model outperforms the other models and allows for $16-28 \%$ additional energy saving in all the considered networks.

\section{Conclusion}

In this paper, we formally define and model the Robust-GreenRE problem. Taking into account the uncertainties of traffic volumes and redundancy elimination rates, the Robust-GreenRE model provides a more accurate evaluation of energy saving for backbone networks. Based on real-life traffic traces, we have shown a significant improvement of energy saving compared with other models. As future work, we shall investigate implementation issues and impacts of Robust-GreenRE model on QoS and fault tolerance.

\section{Acknowledgment}

The authors would like to thank Christelle Caillouet, Frédéric Giroire, Arie M.C.A. Koster, Joanna Moulierac and Martin Tieves for their support. 


\section{References}

[1] A. Altin, E. Amaldi, P. Belotti, M. c. Pinar, Provisioning virtual private networks under traffic uncertainty, Networks 49 (1) (2007) 100-115. doi:10.1002/net.20145.

[2] A. Anand, A. Gupta, A. Akella, S. Seshan, S. Shenker, Packet caches on routers: The implications of universal redundant traffic elimination, SIGCOMM Computer Communication Review 38 (4) (2008) 219-230. doi:10.1145/1402946.1402984.

[3] A. Anand, C. Muthukrishnan, A. Akella, R. Ramjee, Redundancy in network traffic: Findings and implications, in: ACM International Joint Conference on Measurement and Modeling of Computer Systems (SIGMETRICS), ACM, 2009, pp. 37-48. doi: 10.1145/1555349.1555355.

[4] A. Anand, V. Sekar, A. Akella, SmartRE: An architecture for coordinated network-wide redundancy elimination, SIGCOMM Computer Communication Review 39 (4) (2009) 8798. doi:10.1145/1594977.1592580.

[5] J. Baliga, R. Ayre, K. Hinton, W. Sorin, R. Tucker, Energy consumption in optical IP networks, IEEE/OSA Journal of Lightwave Technology 27 (13) (2009) 2391-2403. doi: 10.1109/JLT.2008.2010142.

[6] A. Ben-Tal, L. E. Ghaoui, A. Nemirovski, Robust optimization, Princeton Series in Applied Mathematics, Princeton University Press, 2009.

[7] A. Ben-Tal, A. Nemirovski, Robust optimization - methodology and application, Mathematical Programming 92 (3) (2002) 453-480. doi:10.1007/s101070100286.

[8] D. Bertsimas, M. Sim, Robust discrete optimization and network flows, Mathematical Programming 98 (1-3) (2003) 49 - 71. doi:10.1007/s10107-003-0396-4.

[9] D. Bertsimas, M. Sim, The price of robustness, Operations Research 52 (1) (2004) 35 - 53. doi:10.1287/opre.1030.0065.

[10] D. Bertsimas, D. B. Brown, C. Caramanis, Theory and applications of robust optimization, SIAM Review 53 (3) (2011) 464-501. doi:10.1137/080734510.

[11] A. P. Bianzino, C. Chaudet, F. Larroca, D. Rossi, J. Rougier, Energy-aware routing: A reality check, in: IEEE GLOBECOM Workshop, 2010, pp. 1422-1427. doi:10.1109/ GLOCOMW . 2010.5700172.

[12] BlueCoat: WAN Optimization, http://www.bluecoat.com/.

[13] R. Bolla, F. Davoli, R. Bruschi, K. Christensen, F. Cucchietti, S. Singh, The potential impact of green technologies in next-generation wireline networks: Is there room for energy saving optimization?, IEEE Communications Magazine 49 (8) (2011) 80 - 86. doi:10. 1109/MCOM. 2011.5978419.

[14] J. Chabarek, J. Sommers, P. Barford, C. Estan, D. Tsiang, S. Wright, Power awareness in network design and routing, in: IEEE International Conference on Computer Communications (INFOCOM), 2008, pp. 1130-1138. doi:10.1109/INFOCOM.2008.93.

[15] L. Chiaraviglio, A. Cianfrani, E. Le Rouzic, M. Polverini, Sleep modes effectiveness in backbone networks with limited configurations, Computer Networks 57 (15) (2013) 29312948. doi:10.1016/j. comnet.2013.06.016. 
[16] L. Chiaraviglio, M. Mellia, F. Neri, Minimizing ISP network energy cost: Formulation and solutions, IEEE/ACM Transaction in Networking 20 (2) (2011) 463 - 476. doi: 10.1109/TNET . 2011.2161487.

[17] G. Classen, A. M. C. A. Koster, A. Schmeink, A robust optimisation model and cutting planes for the planning of energy-efficient wireless networks, Computers and Operations Research 40 (1) (2013) 80 - 90. doi:10.1016/j.cor.2012.05.020.

[18] D. Coudert, A. M. C. A. Koster, T. K. Phan, M. Tieves, Robust redundancy elimination for energy-aware routing, in: IEEE International Conference on Green Computing and Communications (GreenCom), 2013, pp. 179-186. doi:10.1109/GreenCom-iThings-CPSCom . 2013.51.

[19] S. Duhovnikov, A. M. C. A. Koster, M. Kutschka, F. Rambach, D. Schupke, Г-robust network design for mixed-line-rate-planning of optical networks, in: IEEE/OSA National Fiber Optic Engineers Conference (OFC/NFOEC), 2013, pp. 1-3.

[20] R. G. Garroppo, S. Giordano, G. Nencioni, M. G. Scutellà, Mixed integer non-linear programming models for green network design, Computers and Operations Research 40 (1) (2013) 273-281. doi:10.1016/j.cor.2012.06.014.

[21] R. G. Garroppo, G. Nencioni, L. Tavanti, M. G. Scutellà, Does traffic consolidation always lead to network energy savings?, IEEE Communications Letters 17 (9) (2013) 1852-1855. doi:10.1109/LCOMM.2013.070913.131244.

[22] F. Giroire, D. Mazauric, J. Moulierac, B. Onfroy, Minimizing routing energy consumption: from theoretical to practical results, in: IEEE/ACM Green Computing and Communications (GreenCom), 2010, pp. 252-259. doi :10.1109/GreenCom-CPSCom.2010.147.

[23] F. Giroire, J. Moulierac, T. K. Phan, F. Roudaut, Minimization of network power consumption with redundancy elimination, in: IFIP TC 6 International Conferences on Networking (NETWORKING), Vol. 7289 of Lecture Notes in Computer Science, Springer, 2012, pp. 247-258. doi:10.1007/978-3-642-30045-5_19.

[24] F. Giroire, J. Moulierac, T. K. Phan, Optimizing rule placement in software-defined networks for energy-aware routing, in: IEEE Global Communications Conference, GLOBECOM, 2014, pp. 2523-2529. 10.1109/GLOCOM.2014.7037187.

[25] Global action plan, http://globalactionplan.org.uk (2007).

[26] T. Grevers, J. Christner, Application Acceleration and WAN Optimization Fundamentals, Fundamentals series, Cisco Press, 2007.

[27] M. Gupta, S. Singh, Greening of the Internet, in: ACM Special Interest Group on Data Communication (SIGCOMM), ACM, 2003, pp. 19-26. doi:10.1145/863955.863959.

[28] W. Van Heddeghem, F. Idzikowski, W. Vereecken, D. Colle, M. Pickavet, P. Demeester, Power consumption modeling in optical multilayer networks, Photonic Network Communications 24 (2) (2012) 86-102. doi:10.1007/s11107-011-0370-7.

[29] IBM ILOG, CPLEX Optimization Studio 12.4, http://www-01.ibm.com/software/ integration/optimization/cplex-optimizer/. 
[30] A. M. C. A. Koster, M. Kutschka, C. Raack, Robust network design: Formulation, valid inequalities, and computations, Networks 61 (2) (2013) 128 - 149. doi:10.1002/net. 21497.

[31] A. M. C. A. Koster, T. K. Phan, M. Tieves, Extended cutset inequalities for the network power consumption problem, in: International Network Optimization Conference (INOC), Vol. 41 of Electronic Notes in Discrete Mathematics, Elsevier, 2013, pp. 69-76. doi: $10.1016 / j$.endm.2013.05.077.

[32] P. Mahadevan, P. Sharma, S. Banerjee, P. Ranganathan, A power benchmarking framework for network devices, in: IFIP TC-6 International Conferences on Networking (NETWORKING), Vol. 5550 of Lecture Notes in Computer Science, Springer, Aachen, Germany, 2009, pp. 795-808. doi:10.1007/978-3-642-01399-7_62.

[33] J. Moulierac, T. K. Phan, Optimizing IGP link weights for energy-efficiency in multi-period traffic matrices, in: Computer Communications, 2015. doi:10.1016/j.comcom.2015.01. 004.

[34] L. Niccolini, G. Iannaccone, S. Ratnasamy, J. Chandrashekar, L. Rizzo, Building a powerproportional software router, in: USENIX Annual Technical Conference, USENIX Association, Boston, MA, USA, 2012, pp. 89-100.

URL http://dl.acm.org/citation.cfm?id=2342821.2342829

[35] S. Orlowski, R. Wessäly, M. Pióro, A. Tomaszewski, SNDlib 1.0 - survivable network design library, Networks 55 (3) (2010) 276-286. doi:10.1002/net.20371.

URL http://sndlib.zib.de

[36] J. Restrepo, C. Gruber, C. Machuca, Energy profile aware routing, in: IEEE International Conference on Communications - Workshop on Green Communications, 2009, pp. 1-5. doi:10.1109/ICCW.2009.5208041.

[37] Y. Song, K. Guo, L. Gao, Redundancy-aware routing with limited resources, in: International Conference on Computer Communications and Networks (ICCCN), 2010, pp. 1-6. doi:10.1109/ICCCN. 2010.5560113.

[38] N. T. Spring, D. Wetherall, A protocol-independent technique for eliminating redundant network traffic, SIGCOMM Computer Communication Review 30 (4) (2000) 87-95. doi: $10.1145 / 347057.347408$.

[39] Steelhead for WAN optimization, http://www.riverbed.com/us/solutions/wan_ optimization/.

[40] M. Zhang, C. Yi, B. Liu, B. Zhang, GreenTE: Power-aware traffic engineering, in: IEEE International Conference on Network Protocols (ICNP), 2010, pp. 21-30. doi:10.1109/ ICNP. 2010.5762751.

[41] E. Zohar, I. Cidon, O. O. Mokryn, The power of prediction: Cloud bandwidth and cost reduction, SIGCOMM Computer Communication Review 41 (4) (2011) 86-97. doi:10. $1145 / 2043164.2018447$. 